\title{
Component separation methods for the PLANCK mission
}

\author{
S. M. Leach ${ }^{1,2}$, J.-F. Cardoso ${ }^{3,4}$, C. Baccigalupi ${ }^{1,2}$, R. B. Barreiro ${ }^{5}$, M. Betoule ${ }^{3}$, J. Bobin ${ }^{6}$, A. Bonaldi ${ }^{7,8}$,
} J. Delabrouille ${ }^{3}$, G. de Zotti ${ }^{1,7}$, C. Dickinson ${ }^{9}$, H. K. Eriksen ${ }^{10,11}$, J. González-Nuevo ${ }^{1}$, F. K. Hansen ${ }^{10,11}$, D. Herranz ${ }^{5}$, M. Le Jeune ${ }^{3}$, M. López-Caniego ${ }^{12}$, E. Martínez-González ${ }^{5}$, M. Massardi ${ }^{1}$, J.-B. Melin ${ }^{13}$, M.-A. Miville-Deschênes ${ }^{14}$, G. Patanchon ${ }^{3}$, S. Prunet ${ }^{15}$, S. Ricciardi ${ }^{7}, 16$, E. Salerno ${ }^{17}$, J. L. Sanz ${ }^{5}$, J.-L. Starck ${ }^{6}$, F. Stivoli ${ }^{1,2}$, V. Stolyarov ${ }^{12}$, R. Stompor ${ }^{3}$, and P. Vielva ${ }^{5}$

1 SISSA - ISAS, Astrophysics Sector, via Beirut 4, 34014 Trieste, Italy e-mail: leach@sissa.it

2 INFN, Sezione di Trieste, via Valerio 2, 34014 Trieste, Italy

3 CNRS \& Université Paris 7, Laboratoire APC, 10 rue A. Domon et L. Duquet, 75205 Paris Cedex 13, France

${ }^{4}$ Laboratoire de Traitement et Communication de l'Information (CNRS and Telecom ParisTech), 46, rue Barrault, 75634 Paris, France

5 Instituto de Física de Cantabria (CSIC-UC), Avda. de los Castros s/n, 39005 Santander, Spain

${ }^{6}$ CEA - Saclay, SEDI/Service d'Astrophysique, 91191 Gif-Sur-Yvette, France

7 INAF - Osservatorio Astronomico di Padova, vicolo dell'Osservatorio 5, 35122 Padova, Italy

8 Dipartimento di Astronomia, vicolo dell'Osservatorio 5, 35122 Padova, Italy

9 Infrared Processing and Analysis Center, California Institute of Technology, M/S 220-6, 1200 E. California Blvd, Pasadena, 91125, USA

${ }^{10}$ Institute of Theoretical Astrophysics, University of Oslo, PO Box 1029 Blindern, 0315 Oslo, Norway

${ }_{11}$ Centre of Mathematics for Applications, University of Oslo, PO Box 1053 Blindern, 0316 Oslo, Norway

12 Astrophysics Group, Cavendish Laboratory, J J Thomson Avenue, Cambridge CB3 0HE, UK

13 DSM/Irfu/SPP, CEA/Saclay, 91191 Gif-sur-Yvette Cedex, France

14 Institut d'Astrophysique Spatiale, Bâtiment 121, 91405 Orsay, France

15 Institut d'Astrophysique de Paris, 98 bis Boulevard Arago, 75014 Paris, France

16 Space Sciences Laboratory, University of California Berkeley, Computational Cosmology Center, Lawrence Berkeley National Laboratory, CA 94720, USA

17 Istituto di Scienza e Technologie dell'Informazione, CNR, Area della ricerca di Pisa, via G. Moruzzi 1, 56124 Pisa, Italy

Received 2 May 2008 / Accepted 17 September 2008

\section{ABSTRACT}

Context. The PLANCK satellite will map the full sky at nine frequencies from 30 to $857 \mathrm{GHz}$. The CMB intensity and polarization that are its prime targets are contaminated by foreground emission.

Aims. The goal of this paper is to compare proposed methods for separating CMB from foregrounds based on their different spectral and spatial characteristics, and to separate the foregrounds into "components" with different physical origins (Galactic synchrotron, free-free and dust emissions; extra-galactic and far-IR point sources; Sunyaev-Zeldovich effect, etc.).

Methods. A component separation challenge has been organised, based on a set of realistically complex simulations of sky emission. Several methods including those based on internal template subtraction, maximum entropy method, parametric method, spatial and harmonic cross correlation methods, and independent component analysis have been tested.

Results. Different methods proved to be effective in cleaning the CMB maps of foreground contamination, in reconstructing maps of diffuse Galactic emissions, and in detecting point sources and thermal Sunyaev-Zeldovich signals. The power spectrum of the residuals is, on the largest scales, four orders of magnitude lower than the input Galaxy power spectrum at the foreground minimum. The CMB power spectrum was accurately recovered up to the sixth acoustic peak. The point source detection limit reaches $100 \mathrm{mJy}$, and about 2300 clusters are detected via the thermal SZ effect on two thirds of the sky. We have found that no single method performs best for all scientific objectives.

Conclusions. We foresee that the final component separation pipeline for PLANCK will involve a combination of methods and iterations between processing steps targeted at different objectives such as diffuse component separation, spectral estimation, and compact source extraction.

Key words. cosmology: cosmic microwave background - methods: data analysis

\section{Introduction}

PLANCK is a European Space Agency space mission whose main objective is to measure the cosmic microwave background $(\mathrm{CMB})$ temperature and polarization anisotropies with high accuracy, high angular resolution and with unprecedented frequency coverage (The Planck Collaboration 2005). In anticipation of the launch, PLANCK is stimulating much research and development into data processing methods that are capable of addressing the ambitious science programme enabled by these multi-frequency observations. It is expected that PLANCK will break new ground in studies of the CMB, of the interstellar 
medium and Galactic emission mechanisms on scales down to a few arcminutes, as well as of the emission from many extragalactic objects.

The processing of such multi-frequency data depends on both the science goals, as well as on the signal to noise regime and on the overall level and complexity of foreground contamination. This observation is borne out by a brief historical perspective on CMB data processing.

An example of the low foreground level and complexity regime is provided by the observations made by Boomerang at 145, 245 and $345 \mathrm{GHz}$ (Masi et al. 2006), which targeted a region of sky with low emission from a single dust foreground. Here the two higher frequency channels acted as foreground monitors for the $145 \mathrm{GHz} \mathrm{CMB}$ deep survey, and were used to estimate that the foreground contamination at $145 \mathrm{GHz}$ was at an rms level of less than $10 \mu \mathrm{K}$ on angular scales of $11.5^{\prime}$ (Table 10, Masi et al. 2006). The $145 \mathrm{GHz}$ CMB maps were then used for the purpose of power spectrum estimation in both temperature and polarization, after masking away a handful of compact sources (Jones et al. 2006). Masi et al. (2006) estimate that the cleanest $40 \%$ of the sky have a level of dust brightness fluctuations similar to those of the Boomerang observations, and that the cleanest $75 \%$ of the sky have brightness fluctuations less than three times larger.

An example of the high foreground level and complexity regime is available with the all-sky observations of the WMAP mission in five frequency channels from 23 to $94 \mathrm{GHz}$ (Bennett et al. 2003a; Hinshaw et al. 2008). In this frequency range, the emission from at least three Galactic components (synchrotron, free-free and dust), as well as contamination by unresolved point sources must be contended with. WMAP also gives a clear example of science goal dependent data processing: CMB maps for use in non-Gaussianity tests are obtained from a noiseweighted sum of frequency maps at differing angular resolution, for which the regions most contaminated by foregrounds are masked (Komatsu et al. 2003); The analysis leading to the WMAP cosmological parameter estimation involves foreground cleaning by template subtraction, masking of the most contaminated $15 \%$ of sky, and subtracting a model of the contribution of unresolved point sources from the $\mathrm{CMB}$ cross power spectra (Hinshaw et al. 2003, 2007). For an improved understanding of galactic emission, the WMAP team have used a number of methods including template fits, internal linear combination (ILC), the maximum entropy method, and the direct pixel-bypixel fitting of an emission model (Gold et al. 2008; Dunkley et al. 2008).

\subsection{Component separation}

Component separation is a catch-all term encompassing any data processing that exploits correlations in observations made at separate frequencies, as well as external constraints and physical modeling, as a means of distinguishing between different physical sources of emission.

PLANCK has a number of different scientific objectives: the primary goal is a cosmological analysis of the CMB, but important secondary goals include obtaining a better understanding of the interstellar medium and Galactic emission, measurement of extragalactic sources of emission and the generation of a Sunyaev-Zeldovich (SZ) cluster catalogue. These planned objectives will lead to a set of data products which the PLANCK consortium is committed to delivering to the wider community some time after the completion of the survey. These data products include maps of the main diffuse emissions and catalogues of extragalactic sources, such as galaxies and clusters of galaxies.

In this context, it is worth remembering that PLANCK is designed to recover the CMB signal at the level of a few microkelvin per resolution element of $5^{\prime}$ (and less than one microkelvin per square degree). Numbers to keep in mind are the rms of CMB smoothed with a beam of $45^{\prime} F W H M$, which is around $70 \mu \mathrm{K}$, while the rms of white noise at the same scale is around $0.7 \mu \mathrm{K}$. This level of sensitivity sets the ultimate goals for data processing - and component separation in particular - if the full scientific potential of PLANCK is to be realised. However, less stringent requirements may be acceptable for statistical analyses such as power spectrum estimation, in particular on large scales where cosmic variance dominates the error of total intensity observations.

\subsection{WG2}

PLANCK is designed to surpass previous CMB experiments in almost every respect. Therefore, a complete and timely exploitation of the data will require methods that improve upon foreground removal via template subtraction and masking. The development and assessment of such methods is coordinated within the PLANCK "Component Separation Working Group" (WG2). Another working group in the PLANCK collaboration, the $C_{\ell}$ temperature and polarization working group (WG3 or "CTP"), investigates other critical data analysis steps, in particular, map-making (Poutanen et al. 2006; Ashdown et al. 2007) and power spectrum estimation.

The present paper reports the results of the WG2 activity in the framework of a component separation challenge using a common set of simulated PLANCK data. ${ }^{1}$ In turn, this exercise provides valuable feedback and validation during the development of the PLANCK Sky Model.

This is the first time, within the PLANCK collaboration, that an extensive comparison of component separation methods has been attempted on simulated data based on models of sky emissions of representative complexity. As will be seen and emphasised throughout this paper, this aspect is critical for a meaningful evaluation of the performance of any separation method. In this respect, the present work significantly improves on the semi-analytical estimates of foreground contamination obtained by Bouchet \& Gispert (1999) for the PLANCK phase A study, as well as on previous work by Tegmark et al. (2000) .

The paper is organised as follows: In Sect. 2 we describe the sky emission model and simulations that were used, and describe the methodology of the Challenge. In Sect. 3 we give an overview of the methods that have been implemented and took part in the analysis. In Sect. 4 we describe the results obtained for $\mathrm{CMB}$ component separation and power spectrum estimation. The results for point sources, SZ and Galactic components are described in Sects. 5 and 6 we present our summary and conclusions. In Appendix A we provide a more detailed description of the methods, their implementation details and their strengths and weaknesses.

\section{The challenge}

The objective of the component separation challenge discussed herein is to assess the readiness of the PLANCK collaboration to

\footnotetext{
1 A similar data challenge has been undertaken in the past in the context of simulated WMAP and sub-orbital CMB data (the WOMBAT challenge; Gawiser et al. 1998).
} 
tackle component separation, based on the analysis of realistically complex simulations. It offers an opportunity for comparing the results from different methods and groups, as well as to develop the expertise, codes, organisation and infrastructure necessary for this task.

This component separation challenge is designed so as to test on realistic simulated data sets, component separation methods and algorithms in a situation as close as possible to what is expected when actual PLANCK data will be analysed. Hence, we assume the availability of a number of ancillary data sets. In particular, we assumed that six-year WMAP observations will be available. Although WMAP is significantly less sensitive than PLANCK, it provides very useful complementary information for the separation of low-frequency Galactic components. This section describes our simulations, the challenge setup, and the evaluation methodology.

\subsection{Sky emission}

Our sky simulations are based on an early development version of the PLANCK Sky Model (PSM, in preparation), a flexible software package developed by PLANCK WG2 for making predictions, simulations and constrained realisations of the microwave sky.

The CMB sky is based on the observed WMAP multipoles up to $\ell=70$, and on a Gaussian realisation assuming the WMAP best-fit $C_{\ell}$ at higher multipoles. It is the same CMB map used by Ashdown et al. (2007).

The Galactic interstellar emission is described by a three component model of the interstellar medium comprising of freefree, synchrotron and dust emissions. The predictions are based on a number of sky templates which have different angular resolution. In order to simulate the sky at PLANCK resolution we have added small scale fluctuations to some of the templates. The procedure used is the one presented in Miville-Deschênes et al. (2007) which allows to increase the fluctuation level as a function of the local brightness and therefore reproduce the nonGaussian properties of the interstellar emission.

Free-free emission is based on the model of Dickinson et al. (2003) assuming an electronic temperature of $7000 \mathrm{~K}$. The spatial structure of the emission is estimated using a $\mathrm{H} \alpha$ template corrected for dust extinction. The $\mathrm{H} \alpha$ map is a combination of the Southern H-Alpha Sky Survey Atlas (SHASSA) and the Wisconsin H-Alpha Mapper (WHAM). The combined map was smoothed to obtain a uniform angular resolution of $1^{\circ}$. For the extinction map we use the $E(B-V)$ all-sky map of Schlegel et al. (1998) which is a combination of a smoothed IRAS $100 \mu \mathrm{m}$ map (with resolution of $6.1^{\prime}$ ) and a map at a few degrees resolution made from DIRBE data to estimate dust temperature and transform the infrared emission in extinction. As mentioned earlier, small scales were added in both templates to match the PLANCK resolution.

Synchrotron emission is based on an extrapolation of the $408 \mathrm{MHz}$ map of Haslam et al. (1982) from which an estimate of the free-free emission was removed. The spectral emission law of the synchrotron is assumed to follow a perfect power law, $T_{b}^{\text {sync }} \propto v^{\beta}$. We use a pixel-dependent spectral index $\beta$ derived from the ratio of the $408 \mathrm{MHz}$ map and the estimate of the synchrotron emission at $23 \mathrm{GHz}$ in the WMAP data obtained by Bennett et al. (2003b) using a Maximum Entropy Method technique. A limitation of this approach is that this synchrotron model also contains any "anomalous" dust correlated emission seen by WMAP at $2 \mathrm{GHz}$.

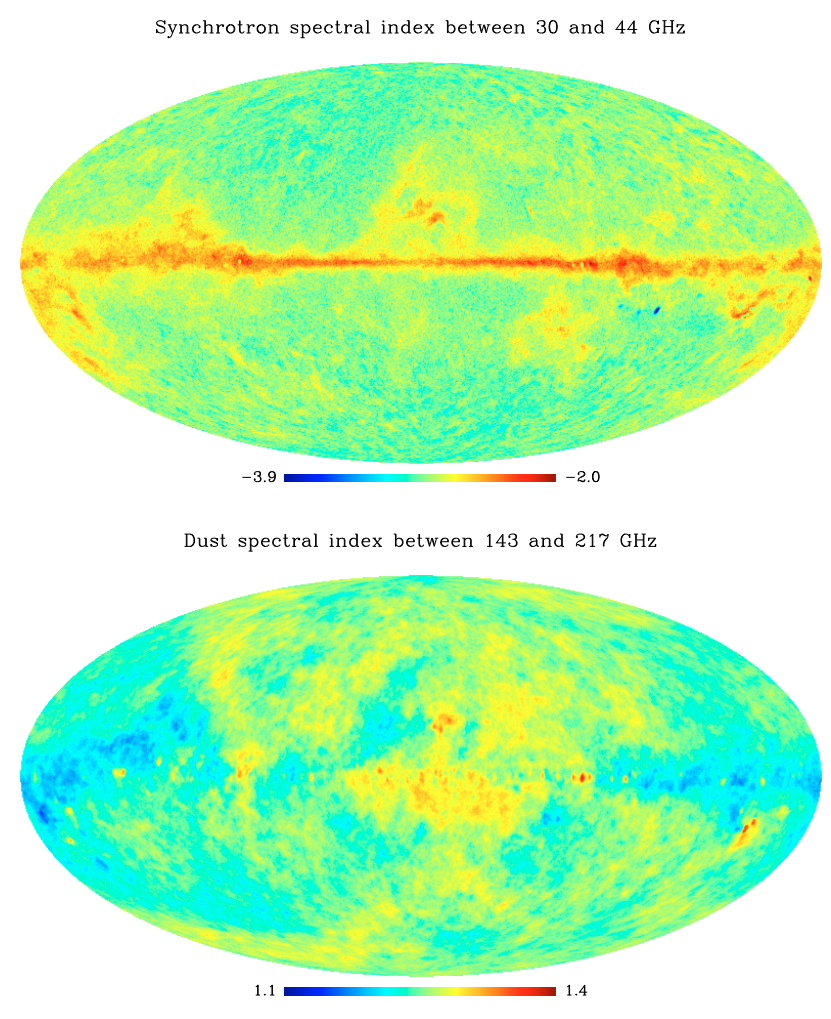

Fig. 1. Synchrotron and dust (effective powerlaw) spectral indices evaluated between 30 and $44 \mathrm{GHz}$, and 143 and $217 \mathrm{GHz}$ respectively (in $\mu \mathrm{K}_{\mathrm{RJ}}$ ). A spatially varying spectral index corresponds to a foreground morphology that varies with frequency.

The thermal emission from interstellar dust is estimated using model 7 of Finkbeiner et al. (1999). This model, fitted to the FIRAS data ( $7^{\circ}$ resolution), makes the hypothesis that each line of sight can be modelled by the sum of the emission from two dust populations, one cold and one hot. Each grain population is in thermal equilibrium with the radiation field and thus has a grey-body spectrum, so that the total dust emission is modelled as

$I_{v} \propto \sum_{i=1}^{2} f_{i} \nu^{\beta_{i}} B_{v}\left(T_{i}\right)$

where $B_{v}\left(T_{i}\right)$ is the Planck function at temperature $T_{i}$. In model 7 the emissivity indices are $\beta_{1}=1.5, \beta_{2}=2.6$, and $f_{1}=0.0309$ and $f_{2}=0.9691$. Once these values are fixed, the dust temperature of the two components is determined using only the ratio of the observations at two wavelengths, $100 \mu \mathrm{m}$ and $240 \mu \mathrm{m}$. For this purpose, we use the $100 / 240 \mu \mathrm{m}$ map ratio published by Finkbeiner et al. (1999). Knowing the temperature and $\beta$ of each dust component at a given position on the sky, we use the $100 \mu \mathrm{m}$ brightness at that position to scale the emission at any frequency using Eq. (1). We emphasise that the emission laws of the latter two components, synchrotron and dust, vary across the sky as shown in Fig. 1. The spectral index of free-free is taken to be uniform on the sky since it only depends on the electronic temperature, taken as a constant here.

Point sources are modelled with two main categories: radio and infra-red. Simulated radio sources are based on the NVSS or SUMSS and GB6 or PMN catalogues. Measured fluxes at 1 and/or $4.85 \mathrm{GHz}$ are extrapolated to PLANCK frequencies assuming a distribution in flat and steep populations. For 
Table 1. Characteristics of PLANCK one year simulations (upper) and WMAP six year simulations ( lower). PLANCK and WMAP hit counts correspond to $1.7^{\prime}$ (Healpix $\left.n_{\text {side }}=2048\right)$ and $6.8^{\prime}\left(n_{\text {side }}=512\right)$ pixels respectively. $N_{\ell}$ is the white noise level calculated from the inhomogeneous distribution of hits.

\begin{tabular}{cccccccccc}
\hline \hline Channel & $30 \mathrm{GHz}$ & $44 \mathrm{GHz}$ & $70 \mathrm{GHz}$ & $100 \mathrm{GHz}$ & $143 \mathrm{GHz}$ & $217 \mathrm{GHz}$ & $353 \mathrm{GHz}$ & $545 \mathrm{GHz}$ & $857 \mathrm{GHz}$ \\
\hline$F W H M[\operatorname{arcmin}]$ & 33 & 24 & 14 & 10 & 7.1 & 5 & 5 & 5 & 5 \\
$\sigma_{\text {hit }}\left[\mu \mathrm{K}_{\mathrm{RJ}}\right]$ & 1030 & 1430 & 2380 & 1250 & 754 & 610 & 425 & 155 & 72 \\
$\sigma_{\text {hit }}\left[\mu \mathrm{K}_{\mathrm{CMB}}\right]$ & 1050 & 1510 & 2700 & 1600 & 1250 & 1820 & 5470 & 24700 & 1130000 \\
Mean; Median hits per pixel & $82 ; 64$ & $170 ; 134$ & $579 ; 455$ & $1010 ; 790$ & $2260 ; 1790$ & $2010 ; 1580$ & $2010 ; 1580$ & $503 ; 396$ & $503 ; 396$ \\
$N_{\ell}^{1 / 2}\left[\mu \mathrm{K}_{\mathrm{CMB}}\right]$ & 0.066 & 0.065 & 0.063 & 0.028 & 0.015 & 0.023 & 0.068 & 0.62 & 28.4 \\
\hline
\end{tabular}

\begin{tabular}{cccccc}
\hline \hline Channel & $23 \mathrm{GHz}(\mathrm{K})$ & $33 \mathrm{GHz}(\mathrm{Ka})$ & $41 \mathrm{GHz}(\mathrm{Q})$ & $61 \mathrm{GHz}(\mathrm{V})$ & $94 \mathrm{GHz}(\mathrm{W})$ \\
\hline$F W H M[\operatorname{arcmin}]$ & 52.8 & 39.6 & 30.6 & 21 & 13.2 \\
$\sigma_{\text {hit }}\left[\mu \mathrm{K}_{\mathrm{RJ}}\right]$ & 1420 & 1420 & 2100 & 2840 & 5210 \\
$\sigma_{\text {hit }}\left[\mu \mathrm{K}_{\mathrm{CMB}}\right]$ & 1440 & 1460 & 2190 & 3120 & 6500 \\
Mean; Median hits per pixel & $878 ; 792$ & $878 ; 790$ & $2198 ; 1889$ & $2956 ; 2577$ & $8873 ; 7714$ \\
$N_{\ell}^{1 / 2}\left[\mu \mathrm{K}_{\mathrm{CMB}}\right]$ & 0.10 & 0.10 & 0.10 & 0.12 & 0.14 \\
\hline
\end{tabular}

each of these two populations, the spectral index is randomly drawn from within a set of values compatible with the typically observed mean and dispersion. Infrared sources are based on the IRAS catalogue, and modelled as dusty galaxies (Serjeant \& Harrison 2005). IRAS coverage gaps were filled by adding simulated sources with a flux distribution consistent with the mean counts. Fainter sources were assumed to be mostly submillimeter bright galaxies, such as those detected by SCUBA surveys. These were modelled following Granato et al. (2004) and assumed to be strongly clustered, with a comoving clustering radius $r_{0} \simeq 8 \mathrm{~h}^{-1} \mathrm{Mpc}$. Since such sources have a very high areal density, they are not simulated individually but make up the sub-mm background.

We also include in the model a map of thermal SZ spectral distortions from galaxy clusters, based on a simulated cluster catalogue drawn from a mass-function compatible with presentday observations and with $\Lambda \mathrm{CDM}$ parameters $\Omega_{\mathrm{m}}=0.3, h=0.7$ and $\sigma_{8}=0.9$ (Colafrancesco et al. 1997; de Zotti et al. 2005).

Component maps are produced at all PLANCK and WMAP central frequencies. They are then co-added and smoothed with Gaussian beams as indicated in Table 1. A total of fourteen monochromatic maps have been simulated.

Finally, inhomogeneous noise is obtained by simulating the hit counts corresponding to one year of continuous observations by PLANCK, using the Level-S simulations tool (Reinecke et al. 2006). An example of a hit count map is shown in the upper panel of Fig. 2. WMAP six year hit counts, obtained from scaling up the observed WMAP three year hit count patterns, are used to generate inhomogeneous noise in the simulated WMAP observations. The rms noise level per hit for both experiments is given in Table 1.

\subsection{Challenge setup}

The simulated data sets were complemented by a set of ancillary data including hitmaps and noise levels, IRAS, $408 \mathrm{MHz}$, and $\mathrm{H} \alpha$ templates, as well as catalogues of known clusters from ROSAT and of known point sources from NVSS, SUMSS, GB6, PMN and IRAS.

The Challenge proceeded first with a blind phase lasting around four months between August and November 2006, when neither the exact prescription used to simulate sky emission from these ancillary data sets, nor maps of each of the input components, were communicated to challenge participants.
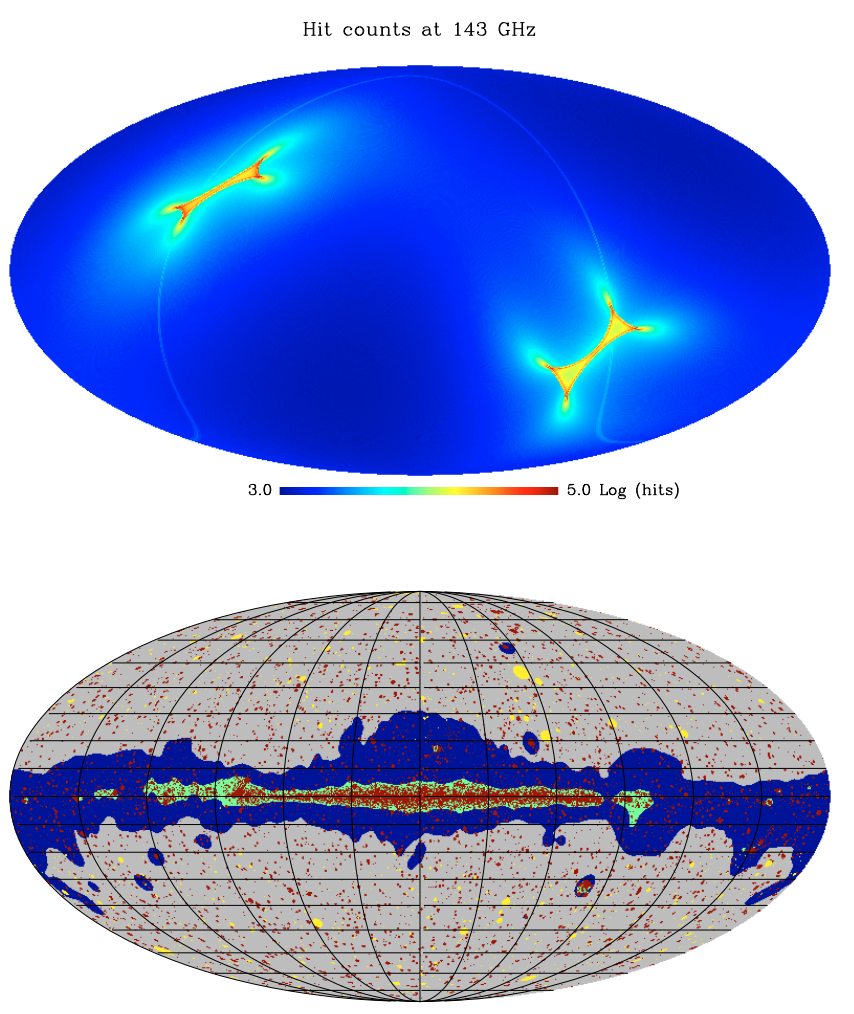

Fig. 2. Upper panel: hit counts for the $143 \mathrm{GHz}$ channel. The inhomogeneities at the ecliptic poles are characteristic of PLANCK's cycloidal scanning strategy. Lower panel: the masking scheme separating the sky in three regions of different foreground contamination. The grey region at high Galactic latitudes is Zone 1 , covering $f_{\text {sky }}=74 \%$. The darker region at lower Galactic latitudes is Zone 2 and covers $f_{\text {sky }}=22 \%$. The remaining region (green) along the Galactic ridge is Zone 3 . The point source mask (red) covers $4 \%$ of Zone 1 . The SZ mask (yellow) cuts detected SZ clusters at Galactic latitudes above 20 degrees, covering $1.4 \%$ of sky.

After this phase and an initial review of the results at the WG2 meeting in Catania in January 2007, the Challenge moved to an open phase lasting from January to June 2007. In this phase the input data-CMB maps and power spectrum, Galactic emission maps, SZ Compton $y$ parameter map, point source 
catalogues and maps, noise realisations - were made available to the participating groups.

All of the results presented here have been obtained after several iterations and improvements of the methods, both during the comparison of the results obtained independently by the various teams, and after the input data was disclosed. Hence, the challenge has permitted significant improvement of most of the methods and algorithms developed within the PLANCK collaboration. The analysis of the Challenge results was led by the simulations team, with involvement and discussion from all participating groups.

\section{Deliverables}

A set of standard deliverables were defined. These included: a CMB map with $1.7^{\prime}$ pixels (Healpix $N_{\text {side }}=2048$ ) together with a corresponding map of estimated errors; the effective beam $F_{\ell}$, which describes the total smoothing of the recovered CMB map due to a combination of instrumental beams and the filtering induced by the component separation process; a set of binned CMB power spectrum estimates (band averages of $\ell(\ell+1) C_{\ell}$ ) and error bars; maps of all the diffuse components identified in the data; catalogues of the infrared and radio sources, and SZ clusters; a map of the SZ Compton $y$ parameter.

\section{Masks}

Different separation methods are likely to perform differently in either foreground-dominated or noise-dominated observations. Also, they may be more or less sensitive to different types of foregrounds. Since the level of foreground contamination varies strongly across the sky, we used a set of standard masks throughout this work, and they are shown in the lower panel of Fig. 2.

The sky is split into three distinct Galactic "Zones": Zone 1 is at high Galactic latitudes and covers $74 \%$ of sky, similar to the WMAP Kp0 mask with smoother edges and small extensions. Zone 2 is at lower Galactic latitudes and covers $22 \%$ of sky. The remaining $4 \%$ of sky is covered by Zone 3, which is similar to the WMAP Kp12 mask.

The point source mask is the product of nine masks, each constructed by excluding a two FWHM region around every source with a flux greater than $200 \mathrm{mJy}$ at the corresponding PLANCK frequency channel. This point source mask covers $4 \%$ of sky in Zone 1. For comparison, the WMAP point source masks of Bennett et al. (2003b) excludes a radius of $0.7^{\circ}$ around almost 700 sources with fluxes greater than $500 \mathrm{mJy}$, covering a total of $2 \%$ of sky.

The SZ mask is constructed by blanking out small circular regions centered on $1625 \mathrm{SZ}$ clusters detected with the needletILC + matched filter method (see Sect. 5.2). For each of them, the diameter of the cut is equal to the virial radius of the corresponding cluster.

\subsection{Comments about the sky emission simulations}

A note of caution about these simulations of sky emission is in order. Although the PSM, as described above, has a considerable amount of sophistication, it still makes some simplifying assumptions - and cannot be expected to describe the full complexity of the real sky. This is a critical issue, as component separation methods are very sensitive to these details. We mention four of them.
First, Galactic emission is modelled with only three components, with no anomalous emission at low frequencies. This affects the spectral behaviour of components in the lower frequency bands below $60 \mathrm{GHz}$ where the anomalous emission is thought to be dominant (Davies et al. 2006; Bonaldi et al. 2007; Miville-Deschênes et al. 2008).

Second, even though variable spectral emission laws are used for synchrotron and dust emission, this is still an idealisation: for the synchrotron, the emission law in each pixel is described by a single spectral index without any steepening. For dust, the emission is modelled as a superposition of two populations, with distinct but fixed temperature and emissivity. These approximations impact component separation, since almost perfect estimation of the relevant parameters of a given foreground emission is possible at frequencies where this foreground dominates, thereby allowing perfect subtraction in the cosmological channels.

Third, it is worth mentioning that only low resolution $\left(\sim 1^{\circ}\right)$ templates are available for synchrotron and free-free emissions. Hence, addition of small-scale power is critical: if such scales were absent from the simulations, but actually significant in the real sky, one might get a false impression that no component separation is needed on small scales. Also, the detection of point sources as well as galaxy clusters would be significantly easier, hence not representative of the actual problem. Here, missing small scale features are simulated using a non-stationary coloured Gaussian random field. Although quite sophisticated, this process can not generate for instance, filamentary or patchy structures known to exist in the real sky.

Fourth, our simulations are somewhat idealised in the sense that we use perfect Gaussian beams, assume no systematic effects, and assume that the noise is uncorrelated from pixel to pixel and from channel to channel. Also, the effect of the finite bandpass of the frequency channels is not taken into account, and we assume that the calibration and zero levels of each channel is perfectly known.

In spite of these simplifications, component separation remains a difficult task with our simulated data because of pixeldependent spectral emission laws for dust and synchrotron, and of the presence of more than a million point sources with different emission laws, of hundreds of thousands of unresolved or extended SZ clusters, and of significant emission from a complex IR background. It is fair to say that this simulated sky is far more complex than anything ever used in similar investigations.

In closing this Section, we show in Fig. 3 the angular power spectra of the basic components for the 70 and $100 \mathrm{GHz}$ channels, close to the foreground emission minimum. The spectra of $\mathrm{CMB}$, noise and thermal SZ are compared to the spectra of the total Galactic emission evaluated at high and low Galactic latitudes, on Zone 1 and 2 respectively. The point source spectra are evaluated in Zone 1, both with and without the brightest sources above 200 mJy masked. Figure 3 shows the obvious impact on CMB studies of masking the most foreground-contaminated regions. It also indicates that there is a significant region of sky, Zone 2, for which Galactic emission and CMB power are comparable. In the following sections, results are evaluated independently in both Zones 1 and 2.

\section{Outline of the methods}

In this section we present a brief overview of the methods that have been used in this challenge. The section is divided in three parts, one for diffuse component separation methods, one for point source extraction, and one for SZ cluster extraction. 


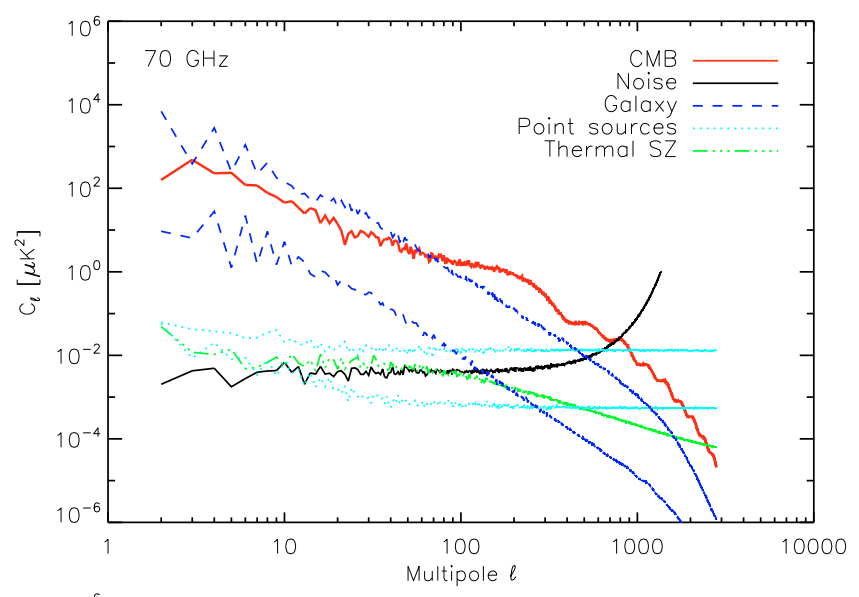

are defined similarly. When this model holds, Eq. (2) becomes

$d_{v}=b_{v} *\left(\sum_{c} A_{v c} s_{c}\right)+n_{v}$.

In simple models, matrix $A$ is constant over the sky; in more complex models, it varies over patches or even from pixel to pixel.

We now briefly describe each of the methods that performed component separation of the CMB (and possibly other diffuse components), and also mention how the CMB angular power spectrum is estimated.

- Gibbs sampling (Commander; Eriksen et al. 2008). The approach of Commander is to fit directly an explicit parametric model of CMB, foregrounds and noise to the antenna temperature of low-resolution map pixels. For the Challenge, Commander was used to analyse the data smoothed to $3^{\circ}$ resolution at each channel with a pixel size of 54' (Healpix $N_{\text {side }}=64$ ). For a given foreground model, Commander provides an exact foreground-marginalised CMB $C_{\ell}$ distributions using the Gibbs (conditional) sampling approach.

- Correlated component analysis (CCA; Bedini et al. 2005). The CCA approach starts with an estimation of the mixing matrix on patches of sky by exploiting spatial correlations in the data, supplemented by constraints from external templates and foreground scaling modeling. The estimated parameters are then used to reconstruct the components by Wiener filtering in the harmonic domain. The $C_{\ell}$ are estimated from the recovered CMB map.

- Independent component analysis (FastICA; Maino et al. 2002). The FastICA method is a popular approach to blind component separation. No assumptions are made about the frequency scaling or mixing matrix. Instead, assuming statistical independence between CMB and foregrounds, the mixing matrix is estimated by maximizing the non-gaussianity of the 1-point distribution function of linear combinations of input data. The inferred mixing matrix is used to invert the linear system of Eq. (4). The $C_{\ell}$ 's are estimated from the recovered CMB map.

\subsection{Diffuse component separation}

The spirit of each method tested on the challenge data is outlined here. A more detailed description, including some details of their implementations and a discussion of their strengths and weaknesses is presented in Appendix A.

First we define some relevant terminology. The data model for a given channel $v$ is

$d_{v}=b_{v} * x_{v}+n_{v}$

where $d_{v}, x_{v}, n_{v}$ are respectively the observation map, the sky emission map and the noise map at frequency $v$ while $b_{v}$ is the instrumental beam of channel $v$, assumed to be Gaussian symmetric, and $*$ denotes convolution on the sphere. The sky emission itself, $x_{v}$, is a superposition of components. Most methods assume (implicitly or explicitly), that it can be written as a linear mixture

$x_{v}=\sum_{c} A_{v c} s_{c}$

where the sum runs over the components. In matrix-vector format, this reads $\boldsymbol{x}=\mathrm{A} \boldsymbol{s}$ where $\mathrm{A}$ is referred to as the "mixing matrix". Vector $\boldsymbol{s}$ is the vector of components. Vectors $\boldsymbol{d}$ and $\boldsymbol{n}$

- Harmonic-space maximum entropy method (FastMEM; Hobson et al. 1998; Stolyarov et al. 2002). The FastMEM method estimates component maps given frequency scaling models and external foreground power spectra (and crosspower spectra) with adjustable prior weight. It is a nonblind, non-linear approach to inverting Eq. (4), which assumes a maximum-entropy prior probability distribution for the underlying components. The $C_{\ell}$ 's are estimated from the recovered $\mathrm{CMB}$ component.

- Generalised morphological component analysis (GMCA; Bobin et al. 2007). Generalised Morphological Component Analysis is a semi-blind source separation method which disentangles the components by assuming that each of them is sparse in a fixed appropriate waveform dictionary such as wavelets. For the Challenge two variants of GMCA were applied: GMCA-blind was optimised for separation of the CMB component, and GMCA-model was optimised for separation of galactic components. The $C_{\ell}$ 's are estimated from the recovered CMB map from the GMCA-blind method.

- Spectral estimation via expectation maximisation (SEVEM; Martínez-González et al. 2003). SEVEM performs component separation in three steps. In a first step, an internal template subtraction is performed in order to obtain foreground-reduced CMB maps in three centre channels 
Table 2. Some characteristics of the diffuse component separation methods used in the challenge.

\begin{tabular}{|c|c|c|c|}
\hline & Channels used & Components modelled & Resources and runtime \\
\hline COMMANDER & WMAP, PLANCK 30-353 GHz, & CMB, dust, sync, FF, mono-,dipoles & 1000 CPU h, 2 day \\
\hline $\mathrm{CCA}$ & PLANCK, Haslam $408 \mathrm{MHz}$ & $\mathrm{CMB}$, dust, sync, FF & 70 CPU h, 1.5 day \\
\hline GMCA & PLANCK, Haslam $408 \mathrm{MHz}$ & CMB, SZ, sync., FF & 1200 CPU h, 6 day \\
\hline FastICA & $143-353 \mathrm{GHz}$ & Two components (CMB and dust) & $21 \mathrm{CPU} \min , 20 \mathrm{~s}$ \\
\hline FastMEM & PLANCK & $\mathrm{CMB}, \mathrm{SZ}$, dust, sync, FF & $256 \mathrm{CPU}$ h, $8 \mathrm{~h}$ \\
\hline SEVEM & PLANCK & $\mathrm{CMB}$ & $30 \mathrm{CPU}$ h, $30 \mathrm{~h}$ \\
\hline SMICA & PLANCK, WMAP & $\mathrm{CMB}, \mathrm{SZ}$, dust, total galaxy & $8 \mathrm{CPU}$ h, $4 \mathrm{~h}$ \\
\hline WI-FIT & 70-217 GHz & CMB & $400 \mathrm{CPU}$ h, 8 h \\
\hline
\end{tabular}

(100-217 GHz). Then the CMB power spectrum is estimated from these maps, via the EM algorithm, assuming a signal plus (correlated) noise model. A final CMB map is obtained using a harmonic Wiener filter on the foreground-reduced maps.

- Spectral matching independent component analysis (SMICA; Delabrouille et al. 2003; Cardoso et al. 2008). The SMICA method estimates model parameters using observation correlations in the harmonic domain (auto- and cross-spectra). The estimated parameters are typically some mixing coefficients and the power spectra of independent components. For the challenge, the correlations between Galactic components are taken into account. The estimated parameters are then used to Wiener-filter the observations to obtain component maps. At small scales the $C_{\ell}$ 's are one of the estimated parameters. At large scales $\ell \leq 100$ the $C_{\ell}$ 's are estimated from a CMB map obtained using the ILC method.

- Wavelet based high resolution fitting of internal templates (WI-FIT; Hansen et al. 2006). The WI-FIT method computes $\mathrm{CMB}$-free foreground plus noise templates from differences of the observations in different channels, and uses those to fit and subtract foregrounds from the CMB dominated channels in wavelet space. The $C_{\ell}$ 's are estimated from the recovered CMB map.

Some characteristics of these methods are summarised in Table 2, which shows the data used, the components modelled and a rough indication of the computational resources required.

Note that many different approaches to diffuse component separation are represented here: blind, non-blind, semi-blind; methods based on linear combinations for foreground extraction; likelihood based methods which estimate parameters of a model of the foregrounds and the CMB; a maximum entropy method; methods based on cross correlations; a method based on sparsity. They also rely on very diverse assumptions and models.

\subsection{Point source extraction}

In the present challenge, point sources are detected in all PLANCK channels independently. Two methods are used, the first based on a new implementation of matched filtering, and the second using the second member of the Mexican Hat Wavelet Family of filters (González-Nuevo et al. 2006). Point sources are detected by thresholding on the filtered maps.

This corresponds to a first step for effective point source detection. It does not exploit any prior information on the position of candidate sources; Such information can be obtained from external catalogues as in López-Caniego et al. (2007), or from detections in other PLANCK channels. Neither does this approach exploit the coherence of the contaminants throughout PLANCK frequencies, nor try to detect point sources jointly in more than one channel. Hence, there is margin for improvement.
- Matched Filter $(M F)$ : the high spatial variability of noise and foreground emission suggests using local filters (for instance on small patches). The sky is divided into 496 overlapping circular regions 12 degrees in diameter. Matched filtering is applied on each patch independently. A local estimate of the power spectrum of the background is obtained from the data themselves by averaging the power in circular frequency bins. A first pass is performed to detect and remove the brightest sources (above 20 $\sigma$ ), in order to reduce the bias in background power estimation and to reduce possible artifacts in the filtered maps. Having removed these bright sources, the $5 \sigma$ level catalogue is obtained by a second application of the whole procedure.

- Mexican Hat Wavelet (MHW2): in a similar way, the sky is divided into 371 square patches. The size of each patch is $14.65 \times 14.65$ square degrees, with a 3 degree overlap among patches. Each patch is then individually filtered with the MHW2. For each patch, the optimal scale of the wavelet is obtained by means of a fast maximization of the wavelet gain factor. This step requires only a straightforward estimation of the variance of the patch, excluding the border and masking any sources above $30 \sigma$. A $5 \sigma$ level catalogue is obtained by simple thresholding in a single step.

\subsection{SZ cluster extraction}

In the present data challenge, we address both the question of building an SZ catalogue, and of making a map of thermal SZ emission.

SZ map: three methods successfully produced SZ maps: ILC in harmonic space, ILC on a needlet frame, and SMICA. For ILC methods, the data are modelled as $\boldsymbol{d}=\boldsymbol{a} s+\boldsymbol{n}$ where $\boldsymbol{d}$ is the vector of observations (nine maps here, using PLANCK data only), $\boldsymbol{a}$ is the SZ spectral signature at all frequencies (a vector with nine entries), $s$ is the component amplitude and $\boldsymbol{n}$ is the noise. The ILC provides an estimator $\widehat{\widehat{S}}_{\mathrm{ILC}}$ of $s$ using

$\widehat{s}_{\mathrm{ILC}}=\frac{\boldsymbol{a}^{t} \widehat{\mathrm{R}}^{-1}}{\boldsymbol{a}^{t} \widehat{\mathrm{R}}^{-1} \boldsymbol{a}} \boldsymbol{d}$

where $\widehat{R}$ is the empirical correlation of the observations, i.e. a $9 \times$ 9 matrix, with entries $R_{v v^{\prime}}$. In practice, the filter is implemented in bands of $\ell$ (ILC in harmonic space) or on subsets of needlet coefficients (ILC in needlet space). The needlet-ILC adapts to the local background to recover the SZ sky.

SZ catalogue: three main methods were used to obtain the cluster catalogue:

- the first one uses a single frequency matched filter (Melin et al. 2006) to extract clusters from the needlet-ILC map; 
- the second one uses SExtractor (Bertin \& Arnouts 1996) to extract clusters from the needlet-ILC map. Then, a single frequency matched filter is used to estimate cluster fluxes;

- the third is a Matched MultiFilter (Herranz et al. 2002), which implements cluster detection using the full set of input observations rather than from an intermediate SZ map. This third method is implemented independently in Saclay and in Santander.

The performance of these four methods is detailed in Table 4. The comparison is done at the same contamination level $(\sim 10 \%)$, which corresponds to $S / N>4.7$ for the needlet-ILC + MF catalogue, $S / N>3.8$ for the needlet-ILC + SExtractor catalogue, $S / N>4.3$ for the Matched Multifilter $(M M F)$ Saclay catalogue and $S / N>4.6$ for the MMF Santander catalogue.

This comparison is being extended to other cluster extraction methods in collaboration with the PLANCK "Clusters and Secondary Anisotropies" working group (WG5). Some improvements are obtained using SExtractor as the extraction tool after the component separation step. There is still some margin for other improvements by increasing the studied area to include lower Galactic latitudes and by combining the SZ extraction methods with $\mathrm{CMB}$ and Galactic extraction methods more intimately.

\section{Results for CMB}

We now turn to the presentation and discussion of the results of the challenge, starting with the CMB component. We evaluate performance based on residual errors at the map and spectral level, and on residual errors at the power spectrum estimation level.

The first point to be made is that all methods have produced CMB maps in Zones 1 and 2. Foreground contamination is barely visible. A small patch representative of CMB reconstruction at intermediate Galactic latitude, is shown in Fig. 9. In the following, we focus on the analysis of the reconstruction error (or residual).

Since each method produces a CMB map at a different resolution, the recovered $\mathrm{CMB}$ maps are compared both against the input CMB sky smoothed only by the $1.7^{\prime}$ pixels, and against a $45^{\prime}$ smoothed version, in order to the emphasise errors at large scales.

\subsection{Map-level residual errors}

Maps of the CMB reconstruction error, with all maps smoothed to a common $45^{\prime}$ resolution, are shown in Fig. 5 for all of the methods (excluding Commander, which produced maps at $3^{\circ}$ resolution). The remaining Galactic contamination is now visible at various levels for most methods, and in particular close to regions with the strongest levels of free-free emission. There is also evidence of contamination by SZ cluster decrements, which are visible as distinct negative sources away from the Galactic plane. As can be seen, significant differences between methods exist.

- At high Galactic latitudes, at this $45^{\prime}$ scale, the lowest contamination is achieved by SMICA, GMCA-BLIND and FastICA.

- In Zone 2, CCA, GMCA-MODEL, and FastMEM seem to filter out Galactic emission best while FastICA and WI-FIT are strongly contaminated.
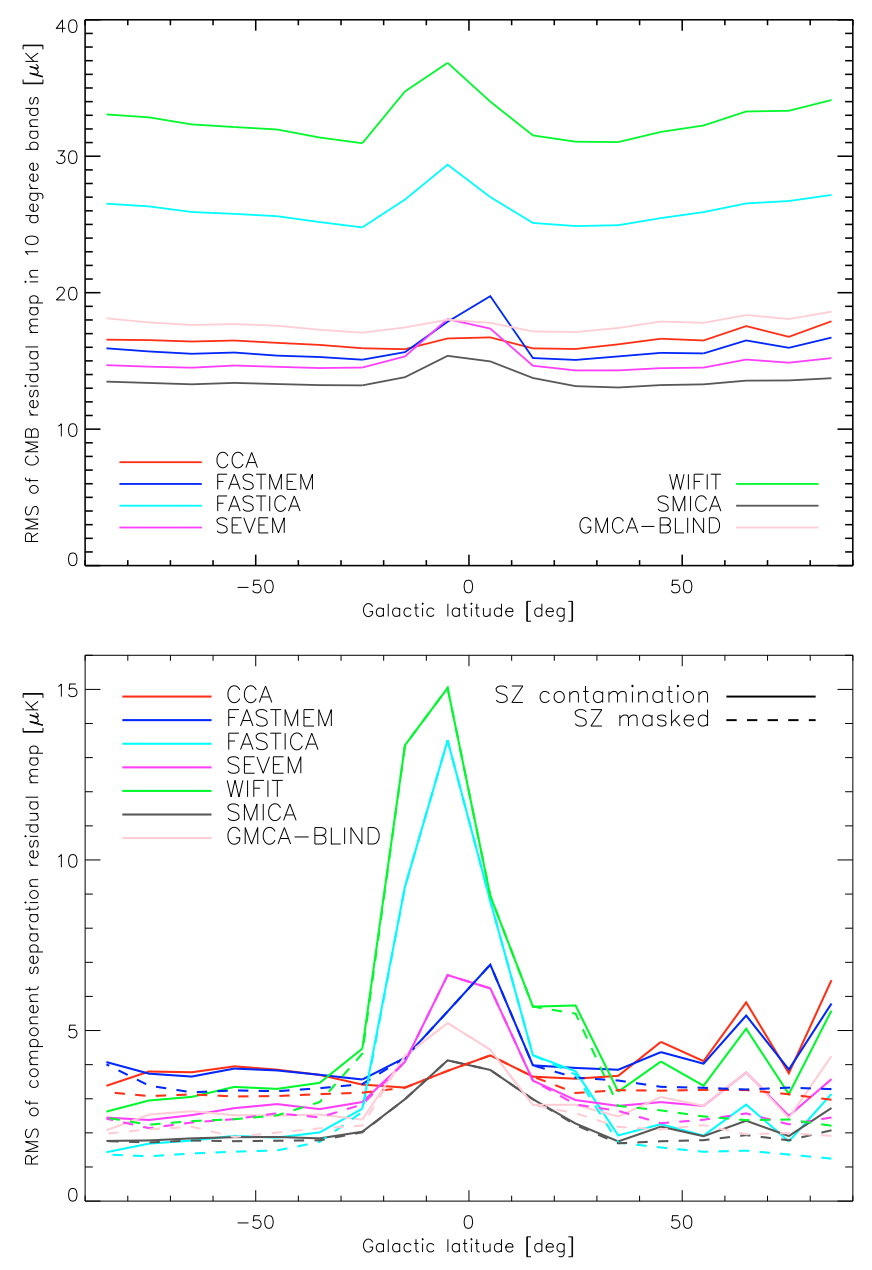

Fig. 4. Upper: rms of the residual error of the CMB map, calculated for each of 18 bands of 10 degrees width in Galactic latitude. For comparison, $\sigma_{\mathrm{CMB}}=104.5 \mu \mathrm{K}$ and $\sigma_{\text {noise }}=29.3 \mu \mathrm{K}$, for the $143 \mathrm{GHz}$ channel alone (1.7' pixels). Lower: rms of this residual map calculated at $45^{\prime}$ resolution. For comparison, $\sigma_{\mathrm{CMB}}\left(45^{\prime}\right)=69.8 \mu \mathrm{K}$ and $\sigma_{\text {noise }}\left(45^{\prime}\right)=0.7 \mu \mathrm{K}$ for the $143 \mathrm{GHz}$ channel. The corresponding residual maps are shown in Fig. 5.

A quantitative measure of the raw residual of the CMB map (reconstructed $\mathrm{CMB}$ minus unsmoothed input $\mathrm{CMB}$ ) is provided by its rms, calculated for 18 zonal bands, each 10 degrees wide in Galactic latitude, excluding pixels in Zone 3 and from the point source mask. The results are shown in the upper panel of Fig. 4. This quantity, denoted $\sigma_{\Delta \mathrm{CMB}}$, gives a measure of the sum of the errors due to residual foreground contamination, noise, as well as from residual CMB (due to non unit response on small scales, for instance). For orientation, we can see that the ensemble of methods span the range $13 \mu \mathrm{K}<\sigma_{\Delta \mathrm{CMB}}<35 \mu \mathrm{K}$, which can be compared with $\sigma_{\mathrm{CMB}}=104.5 \mu \mathrm{K}$ and $\sigma_{\text {noise }}=29.3 \mu \mathrm{K}$, for the $143 \mathrm{GHz}$ channel.

Similarly, the lower panel of Fig. 4 shows the rms of the smoothed residual errors shown in Fig. 5. Depending on the method, the typical level of foreground contamination (plus noise) has an rms from 2 to $5 \mu \mathrm{K}$ on this smoothing scale. For comparison, $\sigma_{\mathrm{CMB}}\left(45^{\prime}\right)=69.8 \mu \mathrm{K}$ and $\sigma_{\text {noise }}\left(45^{\prime}\right)=0.7 \mu \mathrm{K}$ for the $143 \mathrm{GHz}$ channel.

\subsection{Spectral residual errors}

Next we calculate the spectra of the CMB raw residual maps, both on Zone 1 and Zone 2 (high and low Galactic latitudes), 
CMB(out) - CMB(in) (45 arcmin smoothing) using SMICA

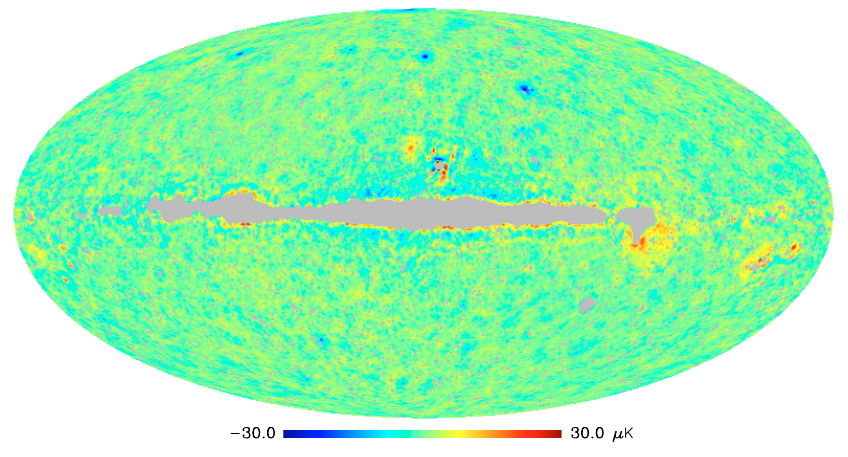

CMB(out) - CMB(in) (45 arcmin smoothing) using CCA

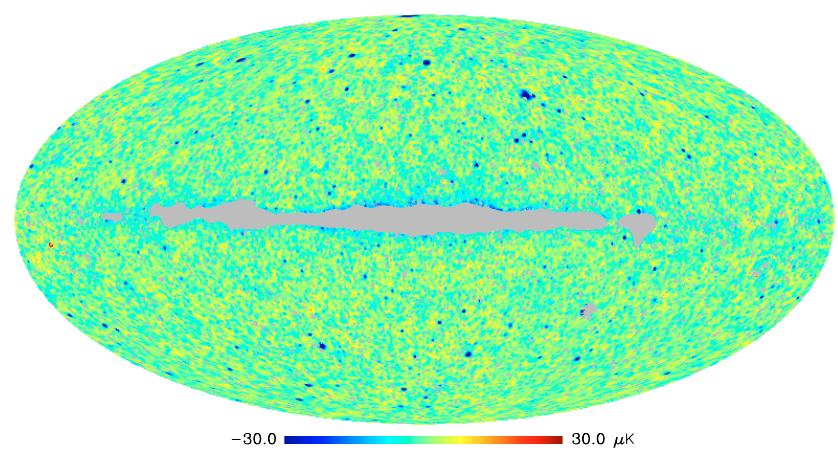

CMB(out) - CMB(in) (45 arcmin smoothing) using FASTICA

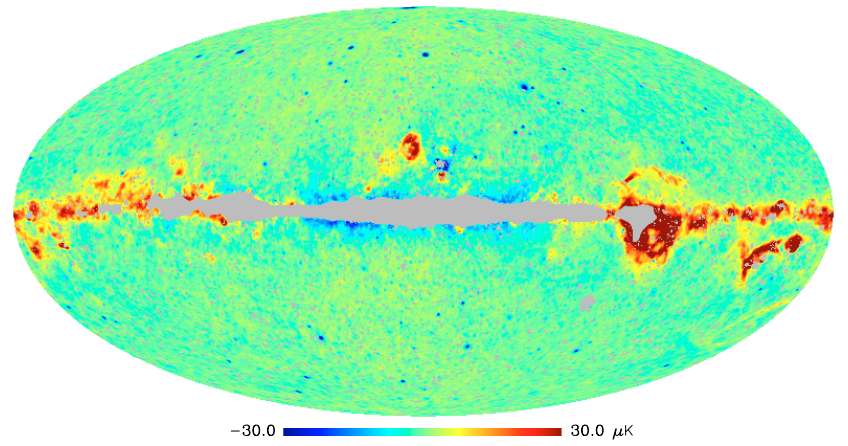

CMB(out) - $\mathrm{CMB}$ (in) (45 arcmin smoothing) using GMCA-MODEL

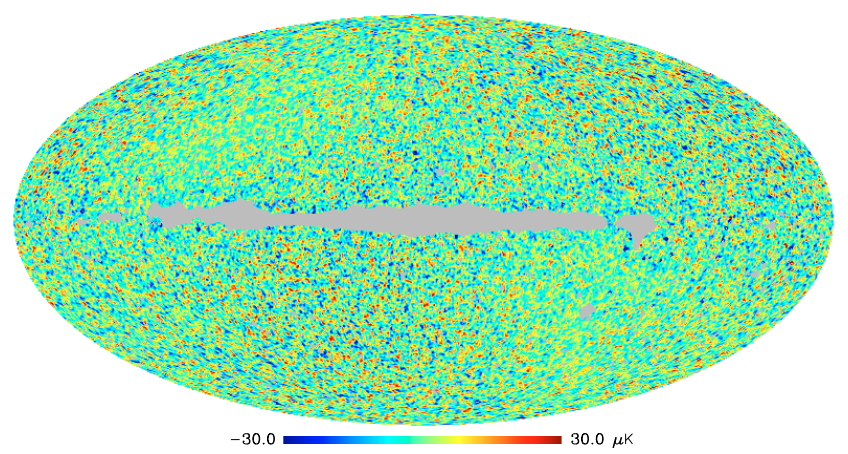

CMB(out) - CMB(in) (45 arcmin smoothing) using SEVEM

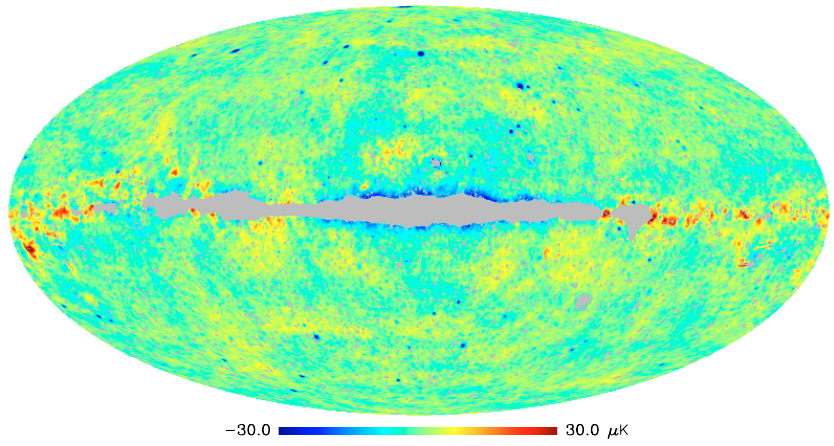

CMB(out) - CMB(in) (45 arcmin smoothing) using GMCA-BLIND

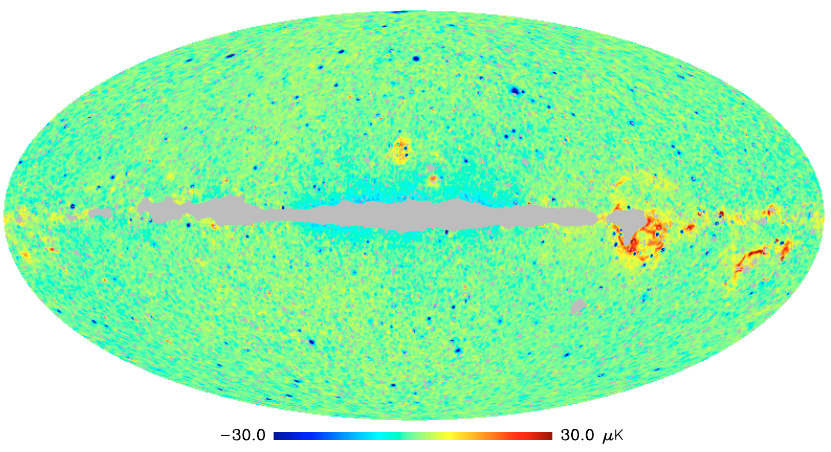

CMB(out) - CMB(jn) (45 arcmin smoothing) using wIFIT

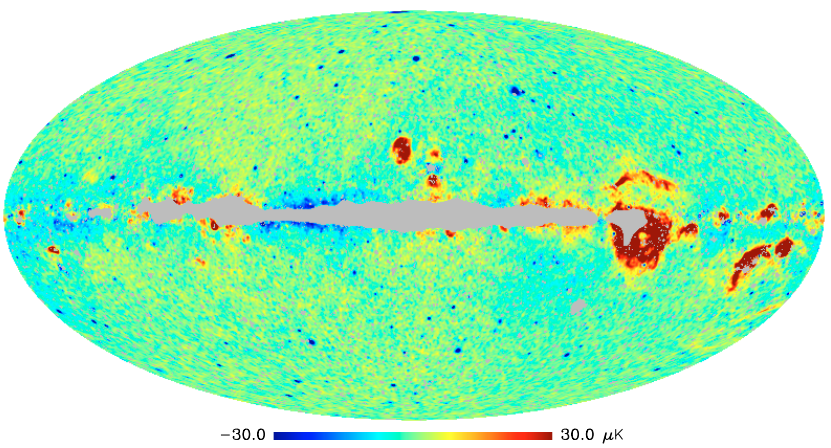

CMB(out) - CMB(in) (45 arcmin smoothing) using FASTMEM

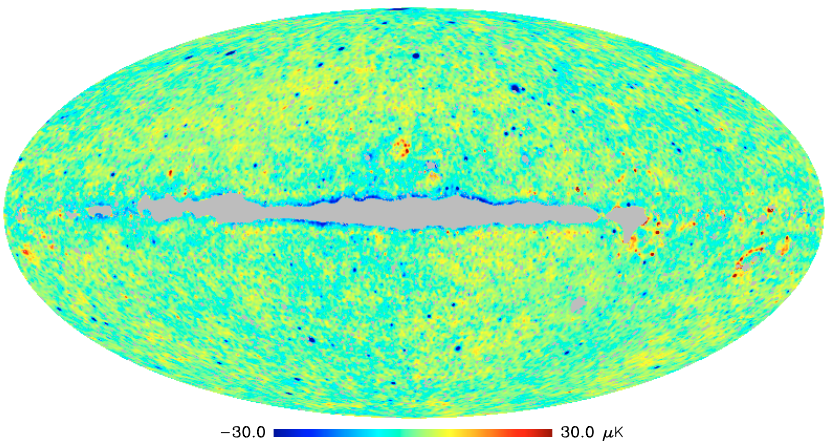

Fig. 5. CMB reconstruction error smoothed at $45^{\prime}$ resolution. These maps are described in Sect. 4.1, and their rms in Galactic latitude strips of $10^{\circ}$ are shown in Fig. 4. Over a large fraction of sky, the typical rms of the residual error is between 2 and $5 \mu \mathrm{K}$, which can be compared with $\sigma_{\mathrm{CMB}}\left(45^{\prime}\right)=69.8 \mu \mathrm{K}$ and $\sigma_{\text {noise }}\left(45^{\prime}\right)=0.7 \mu \mathrm{K}$ for the $143 \mathrm{GHz}$ channel. Some contamination from the galactic components and bright clusters remains. 

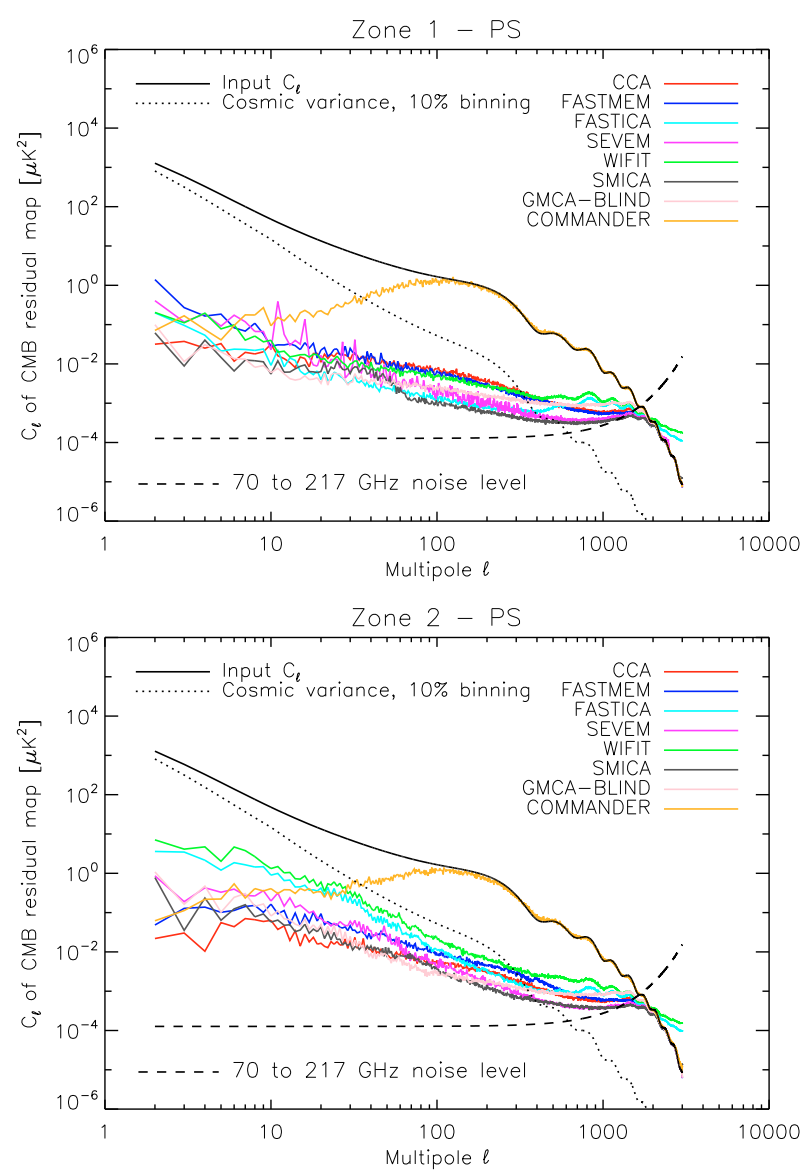

Fig. 6. Spectra of the CMB residual maps, evaluated on Zone 1 (high Galactic latitudes) and Zone 2 (low Galactic latitudes), both regions with point sources masked. Comparison with Fig. 3 shows the extent to which the Galactic contamination has been removed from the $\mathrm{CMB}$ on large angular scales.

with the brightest point sources masked. The results are shown in Fig. 6.

By comparing the spectra of the residuals with the original level of diffuse foreground contamination shown in Fig. 3, we can see that a considerable degree of diffuse foreground cleaning has been attained. There seems however to be a "floor" approached by the ensemble of methods, with a spread of about a factor of ten indicating differences in performance. This floor appears to be mostly free of residual CMB signal which would be visible as acoustic oscillations.

Its overall shape is not white: at high Galactic latitudes the residual spectra bottom out at very roughly $A=0.015 \times$ $\ell^{-0.7} \mu \mathrm{K}^{2}$, while a low Galactic latitudes the spectra bottom out at $A=0.02 \times \ell^{-0.9} \mu \mathrm{K}^{2}$. This limit to the level of residuals is considerably higher than the "foreground-free" noise limit displayed as a dashed line.

It is, however, also significantly lower than the CMB cosmic variance, even with $10 \%$ binning in $\ell$. This comforts us in the impression that component separation is effective enough for CMB power spectrum estimation (discussed next in this paper), although it may remain a limiting issue for other type of CMB science. In particular, it suggests that the component separation residuals, with these channels and the present methods, will dominate the error in PLANCK CMB maps.

Recently Huffenberger et al. (2007) performed a reanalysis of the impact of unresolved point source power in the WMAP three-year data. They found that cosmological parameter constraints are sensitive to the treatment of the unresolved point source power spectrum beyond $\ell=200$ characterised by a white noise level of $A=0.015 \pm 0.005 \mu \mathrm{K}^{2}$. By comparison, the residual foreground contamination obtained in our simulations is as low as $4 \times 10^{-4} \mu \mathrm{K}^{2}$ at $\ell=200$.

\subsection{Power spectrum estimation errors}

Although not the main focus of effort for the Challenge, each group provided their own bandpower estimates of the CMB power spectrum, which in many cases showed obvious acoustic structure out to the sixth or seventh acoustic peak at $\ell \sim 2000$. As an illustration of this result, we show in the upper and middle panels of Fig. 7 the power spectrum estimates from the Commander and SMICA methods respectively.

To make a quantitative estimate of the accuracy of the power spectrum estimates $D_{\ell}$ of $\ell(\ell+1) C_{\ell}$, we calculate the quantity

$\mathrm{FoM}=\frac{\Delta D_{\ell} / D_{\ell}}{\Delta C_{\ell} / C_{\ell}}$

where $\Delta D_{\ell}$ is the bias in the PSE compared to the PSE derived from the input CMB sky, and where $\Delta C_{\ell} / C_{\ell}$ is the expected accuracy of PLANCK, obtained from Eq. (7) below. This figure of merit penalises biases in the power spectrum estimates without taking into account the error bars claimed by each group.

In the absence of foregrounds, an approximate lower bound on the relative standard deviation in estimating the power spectrum is given by

$$
\frac{\Delta C_{\ell}}{C_{\ell}} \simeq \sqrt{\frac{2}{N_{\text {modes }}}}\left(1+\frac{\bar{N}_{\ell}}{C_{\ell}}\right),
$$

where the number $N_{\text {modes }}$ of available modes is

$N_{\text {modes }}=f_{\text {sky }} \sum_{\ell=\ell_{\min }}^{\ell_{\max }}(2 \ell+1)$

where $f_{\text {sky }}$ denotes the fraction of sky coverage. The average noise power spectrum, $\bar{N}_{\ell}$, is obtained from the noise power spectra of the different channels

$$
\begin{aligned}
\bar{N}_{\ell} & =\left(\sum_{v=1}^{N_{\text {chan }}} \frac{B_{v \ell}^{2}}{N_{v \ell}}\right)^{-1}, \\
N_{v \ell} & =\frac{4 \pi \sigma_{\text {hit }}^{2}}{n_{\text {pix }}^{2}} \sum_{p} \frac{1}{n_{\text {hit }}(p)},
\end{aligned}
$$

where $B_{v \ell}$ is the beam window function for channel $v$, and using the calculated values of $N_{v \ell}$ given in Table 1. This theoretical limit Eq. (7) is used below to assess the impact of foregrounds on power spectrum estimation, taking the 70 to $217 \mathrm{GHz}$ channels and assuming the noise levels from Table 1 together with an $f_{\text {sky }}=0.8$.

Ideally, the figure of merit given by Eq. (6) should be much less than one in the cosmic-variance limited regime (i.e. for $\ell \leq$ 500 according to Fig. 6). Significant deviations from zero at low $\ell$ and over \pm 1 at high $\ell$ are indications of significant departures from optimality. We display the FoM Eq. (6), calculated for the PSE of each method in the range $2<\ell<1000$, in the lower panel of Fig. 7.

Focusing first on the range $\ell<20$ we can discern the best performance from Commander, which models the spatial variation of the foreground spectral indices, thus improving the subtraction of foregrounds on large scales. In the range 

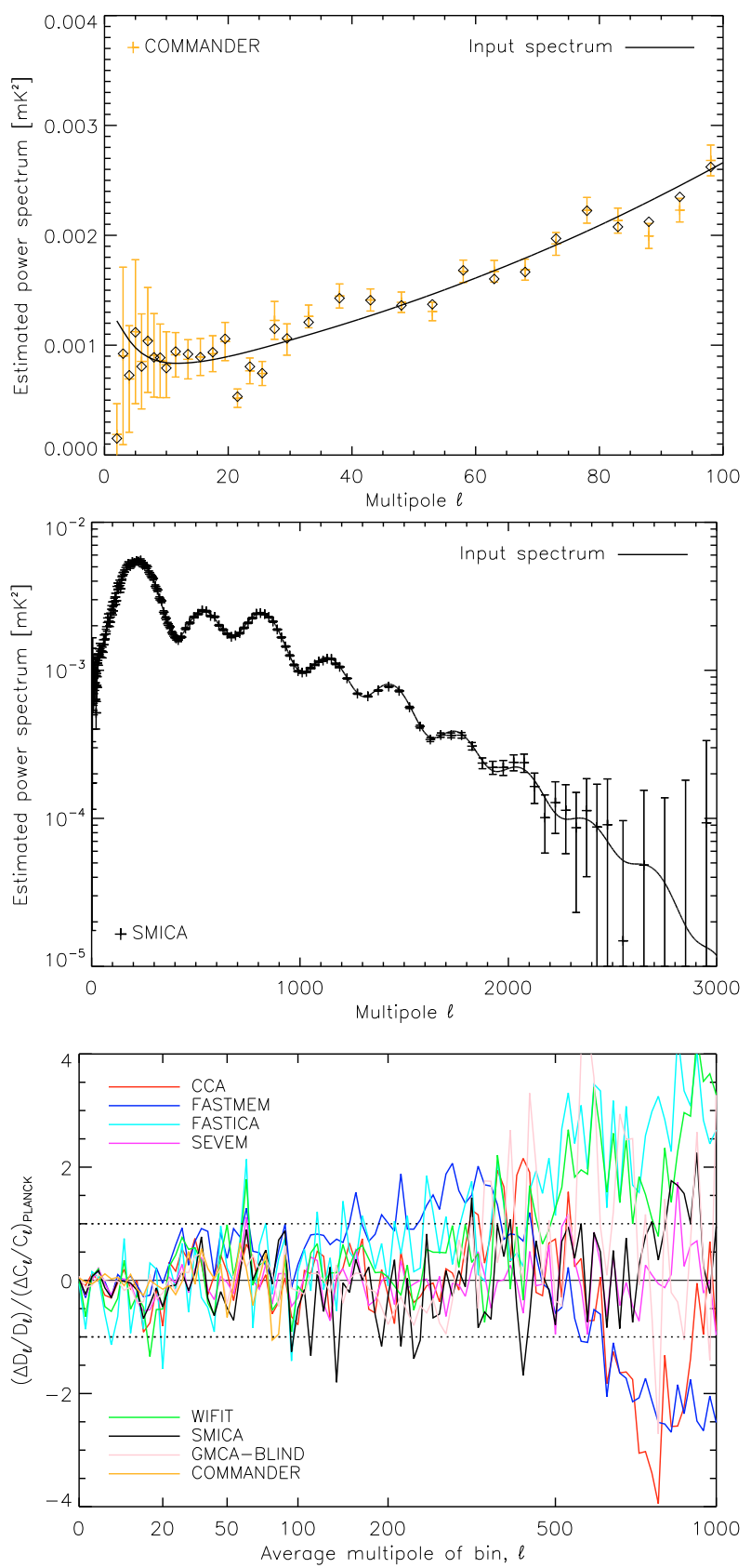

Fig. 7. Upper: power spectrum estimates (PSE) using Commander on large angular scales. The diamonds show the $C_{\ell}$ of the input CMB realisation. Middle: PSE of the recovered CMB map using the SMICA method. Lower: PSE compared with the estimates derived from the input $\mathrm{CMB} C_{\ell}$, and with the expected PLANCK sensitivity, assuming $f_{\text {sky }}=0.8$. Beyond $\ell=500$ biases in the PSE set in in some of the methods.

$20<\ell<500$, SEVEM, specifically designed for an estimate of the CMB power spectrum, performs best among the methods tested on this challenge. Beyond $\ell=500$ we see the best performance from SEVEM and SMICA.

At best, it seems that obtaining PSE with FoM $<1$ is achievable for the multipole range $2<\ell<1000$. Overall though, complete convergence between the results from different methods was not yet achieved.

\subsection{Discussion}

In closing this section on the results for the CMB component, we make some general observations, and attempt to explain some of the differences in these results, as shown in Fig. 5, and with reference to the characteristics of the analyses as detailed in Table 2.

The most significant foreground contamination, where it exists, is associated with regions where free-free emission is most intense, such as the Gum nebula and Orion A and B, and this is most easily visible in the residual maps of FastICA and WIFIT (which also suffers from some dust contamination). Possibly this can be explained by the more limited frequency range of data exploited in these two analyses.

The WMAP data were used in the separation only by Commander and SMICA. In the Commander case, the inclusion of additional low frequency channels (in particular the $23 \mathrm{GHz}$ band) helps to recover the low frequency foregrounds. For SMICA, the use of the WMAP channels was not really mandatory for extracting the CMB, rather they have been used with the objective of developing a pipeline which uses all observations available. WMAP maps are expected to be useful for the extraction of low frequency galactic components. This specific aspect was not investigated further in the present work, as the simulations used here (which do not include any anomalous dust emission) are not really adequate to address this problem meaningfully.

\section{Results for other components}

\subsection{Point sources}

Additional efforts have been directed towards producing a catalogue of point sources, a catalogue of SZ clusters and maps of the thermal SZ effect and Galactic components.

The detection of point sources is both an objective of PLANCK component separation (for the production of the PLANCK early release compact source catalogue (ERCSC) and of the final point source catalogue), and also a necessity for CMB science, to evaluate and subtract the contamination of $\mathrm{CMB}$ maps and power spectra by this population of astrophysical objects (Wright et al. 2008; González-Nuevo et al. 2008).

The matched filter and the Mexican hat wavelet 2 have advantages and drawbacks. In principle, the matched filter is the optimal linear filter. However, it often suffers from inaccurate estimation of the required correlation matrix of the contaminants, and from the difficulty to adapt the filter to the local contamination conditions. On the data from the present challenge, this resulted in excessive contamination of the point source catalogue by small scale Galactic emission, mainly dust at high frequencies.

Table 3 summarises the PS detection achieved by these methods. We found that the Mexican hat wavelet 2 and the matched filter performed similarly in most of the frequency channels, and complement each other in the others. Performance depends on the implementation details, and on properties of the other foregrounds.

It should be noted that, for all channels, the $5 \sigma$ detection limit is somewhat above what would be expected from (unfiltered) noise alone (by a factor 1.33 for the best case, $44 \mathrm{GHz}$, to 4.8 for the worst case, $857 \mathrm{GHz}$ ). This is essentially due to the impact of other foregrounds and the CMB, as well as confusion with other sources. In particular, this effect is more evident at 545 and $857 \mathrm{GHz}$, due to high dust contamination but also due to the confusion with the highly correlated population of SCUBA 
Table 3. Results of point source detection on the present data challenge.

\begin{tabular}{cccccccccc}
\hline \hline Channel & $30 \mathrm{GHz}$ & $44 \mathrm{GHz}$ & $70 \mathrm{GHz}$ & $100 \mathrm{GHz}$ & $143 \mathrm{GHz}$ & $217 \mathrm{GHz}$ & $353 \mathrm{GHz}$ & $545 \mathrm{GHz}$ & $857 \mathrm{GHz}$ \\
\hline MF: flux limit [mJy] (5\% cont.) & 420 & 430 & 360 & 220 & 130 & 100 & 190 & 890 & 2490 \\
Detections & 655 & 591 & 623 & 1103 & 2264 & 2597 & 1994 & 1200 & 1132 \\
\hline MHW2: flux limit [mJy] (5\% cont.) & 395 & 450 & 380 & 250 & 140 & 120 & 230 & 430 & 2160 \\
Detections & 762 & 621 & 599 & 1065 & 2072 & 2203 & 1650 & 1832 & 1259 \\
\hline
\end{tabular}

Table 4. Performance of the SZ cluster detection methods. The table gives the absolute number of detection for $|b|>20^{\circ}$.

\begin{tabular}{cccc}
\hline \hline Method & Detections & False & Reliability \\
\hline Needlet-ILC + SExt. & 2564 & 225 & $91 \%$ \\
Needlet-ILC + MF & 1804 & 179 & $90 \%$ \\
MMF Saclay & 1803 & 178 & $90 \%$ \\
MMF IFCA & 1535 & 144 & $91 \%$ \\
\hline
\end{tabular}

\subsection{Galactic components}

For the Challenge, a number of methods were applied for separating out Galactic components. Table 2 lists which Galactic components were obtained by the different methods. Five groups have attempted to separate a high frequency dust-like component. Four groups have attempted separation of synchrotron and free-free at low frequencies.

We compared the reconstructed component maps with their counterpart input maps, both in terms of the absolute residual error and in terms of the relative error. Both these measures are computed after removing the best-fit monopole and dipole from the residual error map (fitted when excluding a region $\pm 30^{\circ}$ in Galactic latitude). We then defined a figure of merit $f_{20 \%}$, which corresponds to the fraction of sky where the foreground amplitude is reconstructed with a relative error of less than $20 \%$.

The main results can be summarised as follows: The dust component was the best reconstructed component with an $f_{20 \%} \simeq$ 0.7 for all methods. The relative error typically becomes largest at the higher galactic latitudes where the dust emission is faintest. The synchrotron component was reconstructed with an $f_{20 \%} \simeq 0.3-0.5$, with Commander achieving the best results at $3^{\circ}$ resolution, but with noticeable errors along the galactic ridge where, in our simulations, the synchrotron spectral index flattens off. Free-free emission is detected and identified in regions such as the Gum Nebula, Orion A and B, and the Ophiucus complex. However, the reconstruction of the free-free emission at low Galactic latitudes needs improving. On the other hand, the total Galactic emission (free-free plus synchrotron) at lowfrequencies is better reconstructed, with $f_{20 \%} \simeq 0.5-0.8$, with the best results from Commander.

In Fig. 9, we show for illustration the recovered total Galactic emission at $23 \mathrm{GHz}$ from Commander, the dust emission at $143 \mathrm{GHz}$ from FastICA and, for comparison, the recovered CMB from SMICA on the same patch.

\section{Summary and conclusions}

In this paper we have described a CMB component separation Challenge based on a set of realistic simulations of the PLANCK satellite mission. The simulated data were based on a development version of the PLANCK Sky Model, and included the foreground emission from a three component Galactic model of freefree, synchrotron and dust, as well as radio and infra-red sources, the infra-red background, the SZ effect and PLANCK-like inhomogeneous noise. We have cautioned that the simulations, while complex, still relied on some simplifying assumptions, as discussed in Sect. 2.3. Thus, there is no guarantee that the priors and data models that yielded the best separation on simulations will work equally well on the PLANCK dataset. While this may seem to undercut the main purpose of this paper, we are simply acknowledging that we cannot anticipate in full detail what the PLANCK component separation pipeline will look like and how effective it will be, based on an analysis of present-day simulations. In spite of this, it is clear that methods performing better on the simulated data have in general better chances to work better on the real sky. 
ILC needlet SZ

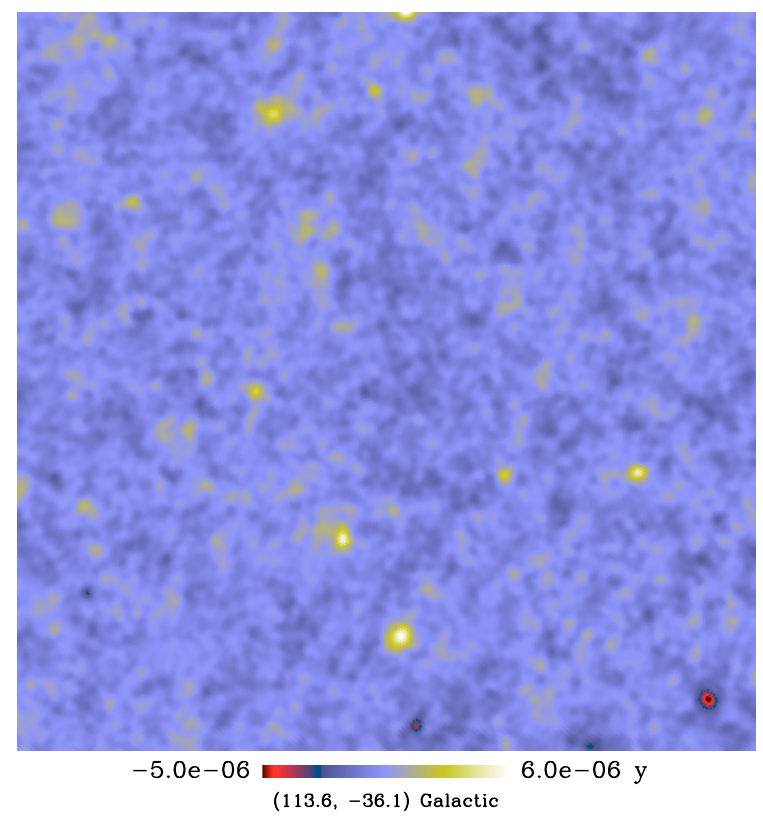

Input SZ map

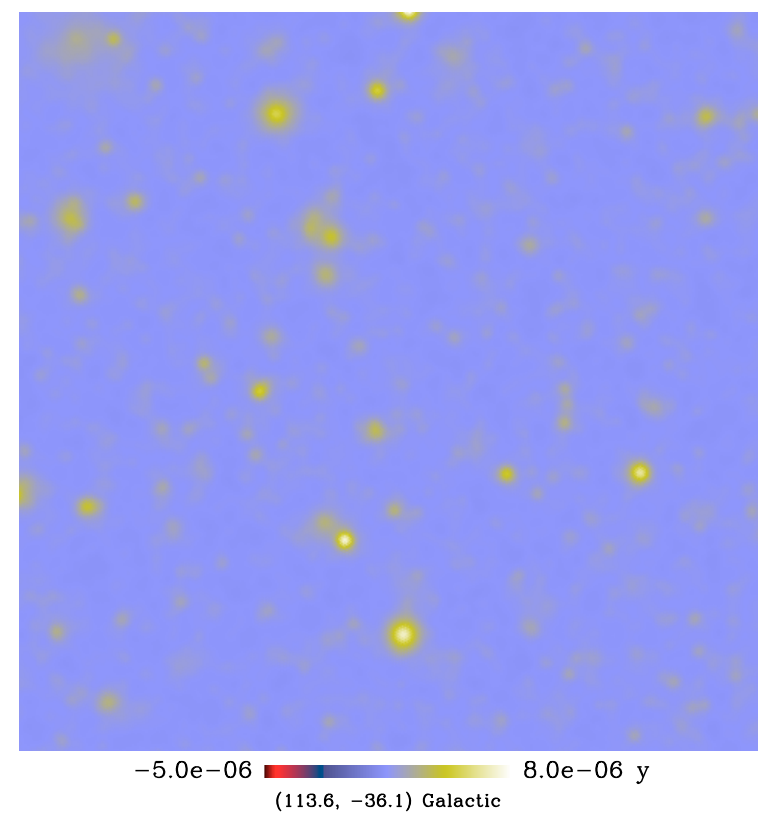

Fig. 8. Patch of the recovered needlet-ILC SZ map and input SZ map. For easier comparison of the two maps $(12.5 \times 12.5 \mathrm{deg})$, the input SZ map has been filtered to the same resolution as the output.

As a combined set of tools, the component separation methods developed and tested in this work offer very different ways to address the component separation problem and so comparable performance between different tools, when achieved, provides confidence in the conclusions of this work against some of the simplifications used in the model for simulating the sky emission.

We found that the recovered CMB maps were clean on large scales, in the sense that the rms of the residual contamination was much less than the cosmic variance: at best the rms residual of the cleaned CMB maps was of the order of $2 \mu \mathrm{K}$ across the sky on a smoothing scale of $45^{\prime}$, with a spectral distribution described by $A=0.015 \times \ell^{-0.7} \mu \mathrm{K}^{2}$ and $A=0.02 \times \ell^{-0.9} \mu \mathrm{K}^{2}$ at high and low Galactic latitudes respectively. The effectiveness of the foreground removal is illustrated by comparing the input foreground power spectra of Fig. 3 with the residuals shown in Fig. 6. The two panels of the latter figure show that, with few exceptions, the methods manage to clean the low Galactic latitude Zone 2, where the foreground contamination is high, almost as well as they do for the high Galactic latitude Zone 1, where the CMB dominates at the frequencies near the foreground minimum. The amplitude of the power spectrum of residuals is, on the largest scales, four orders of magnitude lower than that of the input Galaxy power spectrum at the foreground minimum. This means that the CMB map could be recovered, at least by some methods, over the whole sky except for a sky cut at the 5 percent level (see Fig. 4). The CMB power spectrum was accurately recovered up to the sixth peak.

As detailed in Table 2, the outputs of the methods were diverse. While all have produced a CMB map, only a subset of them were used to obtain maps of individual diffuse Galactic emissions. Five (Commander, CCA, FastICA, FastMEM, SMICA) reconstructed thermal dust emission maps at high frequencies, and another five (Commander, CCA,
FastMEM, GMCA, SMICA) yielded a map of the low-frequency Galactic emissions (synchrotron and free-free).

It is not surprising that the dust component was more easily reconstructed because it is mapped over a larger frequency range, and benefits from observations at high frequencies where it dominates over all other emissions, except the IR background at high Galactic latitudes. Moreover at high frequencies the noise level is lower and the angular resolution is better. Low frequency Galactic foregrounds suffer from more confusion, with a mixture of several components observed in only few channels, at lower resolution.

The relative errors of the reconstructed foreground maps are larger at high Galactic latitudes where the foregrounds are fainter. We have defined a figure of merit $f_{20 \%}$, which corresponds to the fraction of sky where the amplitude of each galactic component has been reconstructed with a relative error of less than $20 \%$. For most methods, $f_{20 \%} \simeq 70 \%$ was achieved for the dust component, while $f_{20 \%} \simeq 50 \%$ was achieved for the radio emission, increasing to $80 \%$ if component separation is performed at a relatively low resolution of $3^{\circ}$. Clearly, there is ample room and need for improvement in this area.

The flux limits for extragalactic point source detection are minimum at 143 and $217 \mathrm{GHz}$, where they reach $\simeq 100 \mathrm{mJy}$. About 1000 radio sources and about 2600 far-IR sources are detected over about $67 \%$ of the sky $\left(|b|>20^{\circ}\right)$. Over the same region of the sky, the best methods recover about 2300 clusters.

In closing, we list areas where work is in progress and improvements are expected: The sky model is being upgraded to include the anomalous emission component and polarization. We are in the process of integrating point source and SZ extraction algorithms together with the diffuse component separation algorithms into a single component separation pipeline. This is expected, on one side, to decrease the contamination of CMB maps on small angular scales, where point and compact sources (including SZ effects) dominate and, on the other side, 


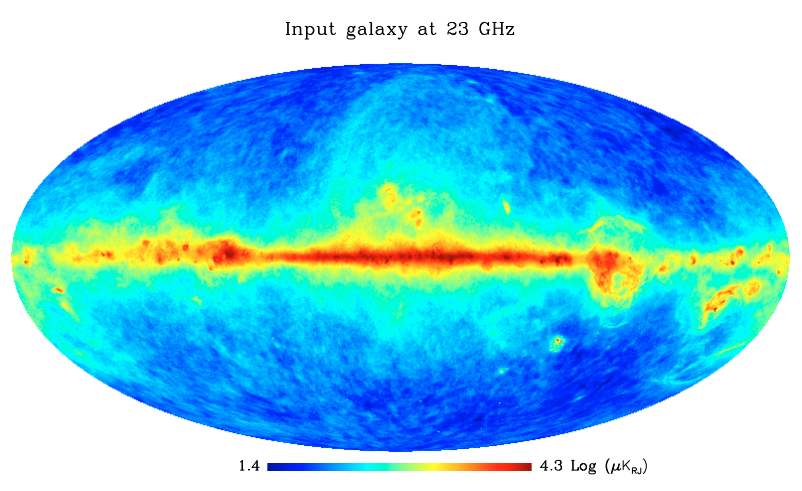

Input dust at $143 \mathrm{GHz}$

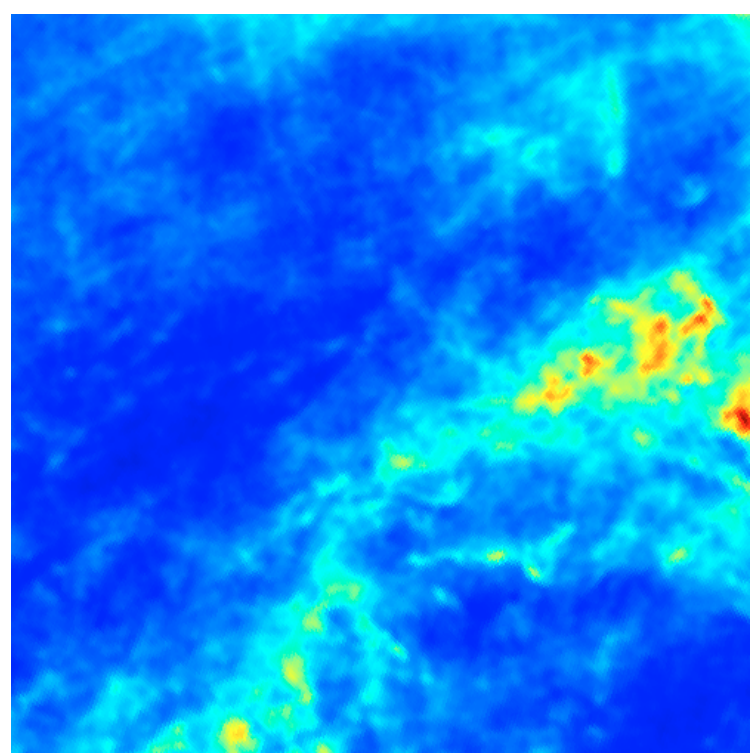

$0.0 \underset{(113.6,-38.1) \text { Galactic }}{ } 55.4 \mu \mathrm{K}_{\mathrm{RJ}}$

Input CMB map

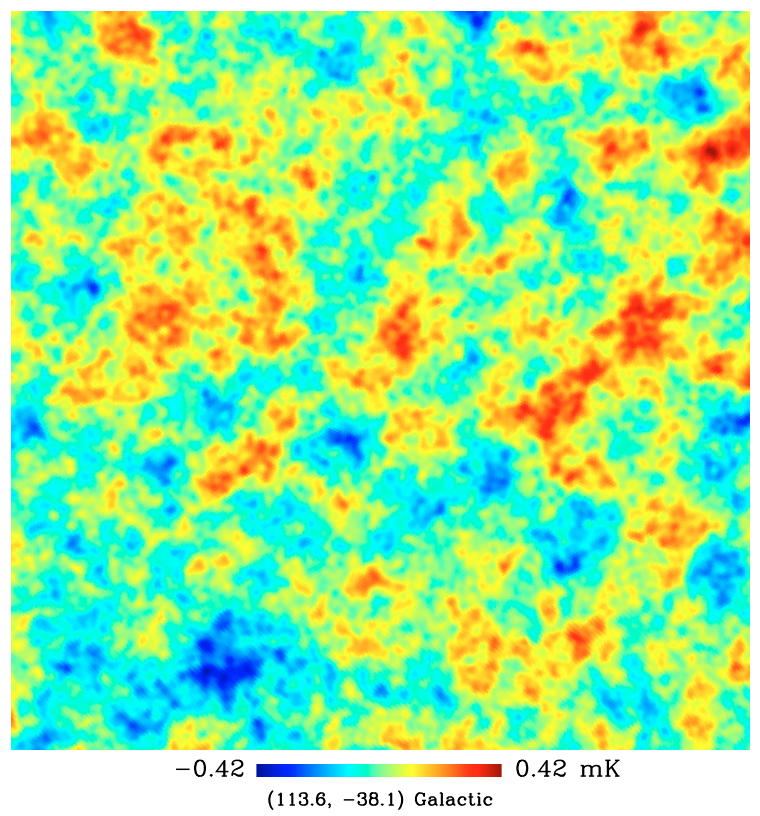

Commander galaxy at $23 \mathrm{GHz}$

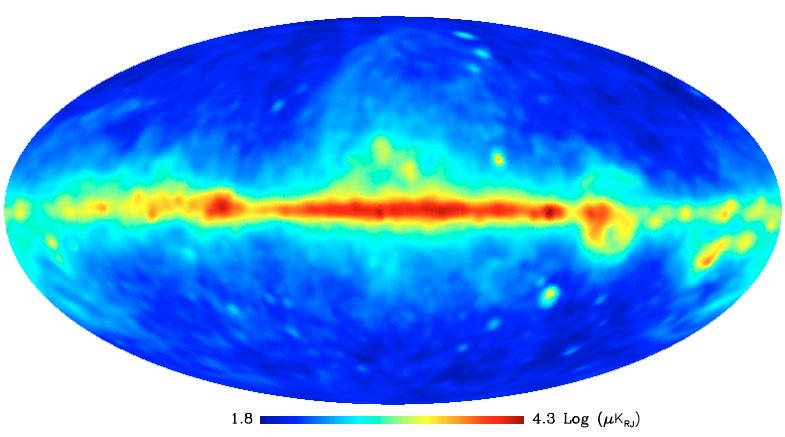

FastICA dust at $143 \mathrm{GHz}$

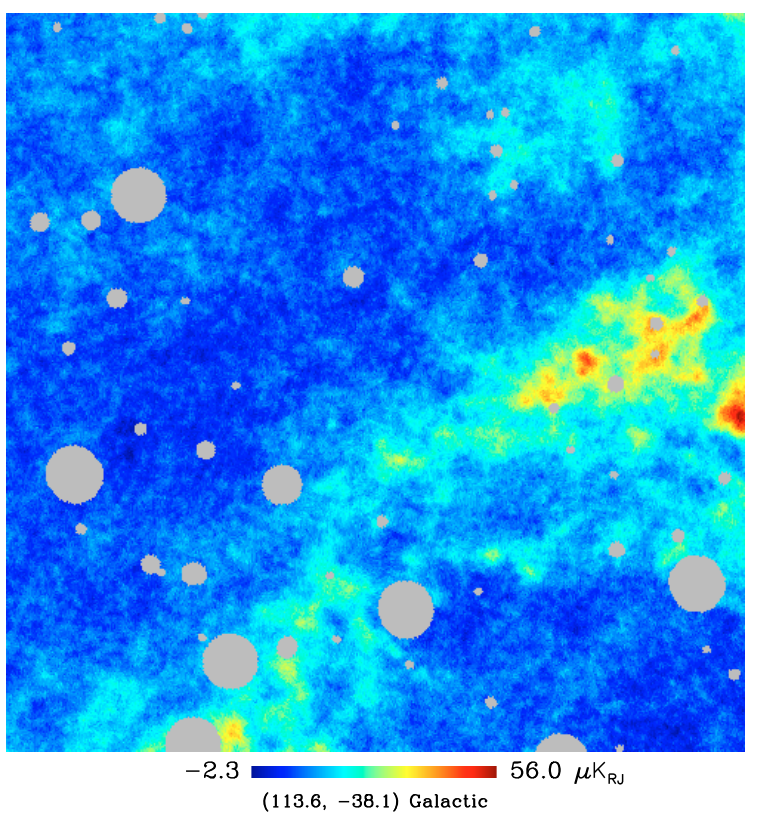

SMICA CMB

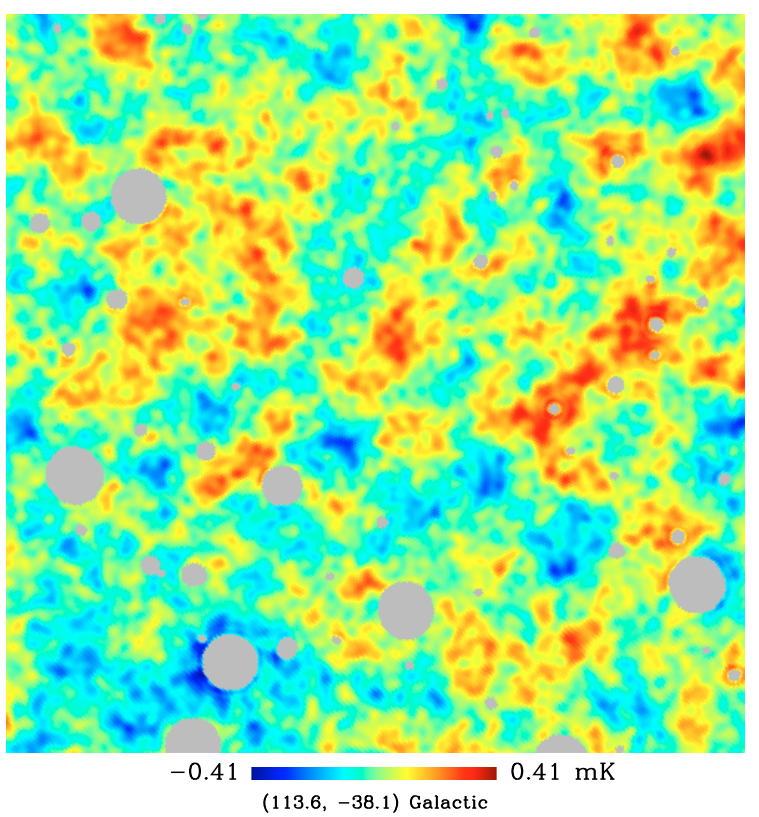

Fig. 9. Example input and recovered total galaxy emission at $23 \mathrm{GHz}$, dust at $143 \mathrm{GHz}$ and $\mathrm{CMB}$ components. 
to achieve a more efficient point and compact source extraction. Assessing this in more detail is part of WG2 work plans for the near future. The SEVEM foreground cleaning step now operates in wavelet space which allows for improved, scale-by-scale removal of foregrounds. In addition the recovery of the power spectrum estimates and error bars at the highest multipoles has been improved by reducing the cross-correlation between modes through the use of an apodised mask. For Commander, work is currently ongoing to extend the foreground sampler to multiresolution experiments. CCA is being upgraded to fully exploit the estimated spatially-varying spectral indices in the source reconstruction step; SMICA is being improved to model unresolved point source power. The FastICA algorithm is being improved to handle data with a wider frequency coverage. The GMCA framework is being extended to perform a joint separation and deconvolution of the components.

Acknowledgements. The work reported in this paper was carried out by Working Group 2 of the PLANCK Collaboration. PLANCK is a mission of the European Space Agency. The Italian group were supported in part by ASI (contract Planck LFI Phase E2 Activity). The US PLANCK Project is supported by the NASA Science Mission Directorate. The ADAMIS team at APC has been partly supported by the Astro-Map and Cosmostat ACI grants of the French ministry of research, for the development of innovative CMB data analysis methods. The IFCA team acknowledges partial financial support from the Spanish project AYA200768058-C03-02. This research used resources of the National Energy Research Scientific Computing Center, which is supported by the Office of Science of the US Department of Energy under Contract No. DE-AC02-05CH11231. D.H., J.L.S. and E.S. would like to acknowledge partial financial support from joint CNR-CSIC research project 2006-IT-0037. R.S. acknowledges support of the E.C. Marie Curie IR Grant (mirg-ct-2006-036614). C.B. was partly supported by the NASA LTSA Grant NNG04CG90G. S.M.L. would like to thank the kind hospitality of the APC, Paris for a research visit where part of this work was completed. We thank Carlo Burigana for a careful reading of the manuscript. We acknowledge the use of the Legacy Archive for Microwave Background Data Analysis (LAMBDA). Support for LAMBDA is provided by the NASA Office of Space Science. The results in this paper have been derived using the HEALPix package (Górski et al. 2005).

\section{Appendix A: Description of methods}

\section{A.1. Commander}

"Commander" is an implementation of the CMB and foregrounds Gibbs sampler most recently described by Eriksen et al. (2008); This algorithm maps out the joint CMB-foreground probability distribution, or "posterior distribution", by sampling. The target posterior distribution may be written in terms of the likelihood and prior using Bayes' theorem,

$$
\operatorname{Pr}\left(\boldsymbol{s}, C_{\ell}, \theta_{\mathrm{fg}} \mid \boldsymbol{d}\right)=\mathcal{L}\left(\boldsymbol{d} \mid \boldsymbol{s}, \theta_{\mathrm{fg}}\right) \operatorname{Pr}\left(\boldsymbol{s} \mid C_{\ell}\right) \operatorname{Pr}\left(C_{\ell}\right) \operatorname{Pr}\left(\theta_{\mathrm{fg}}\right) .
$$

Here $\theta_{\mathrm{fg}}$ is the collection of all parameters required to describe the non-cosmological foregrounds. Since the noise is assumed to be Gaussian, the likelihood is simply given by the $\chi^{2}$.

In the current analysis, the foregrounds are modelled by a sum of synchrotron, free-free and thermal dust emission, and free monopole and dipoles at each frequency band. The thermal dust component is approximated by a single-component modified blackbody with a fixed dust temperature $T_{\mathrm{d}}=21 \mathrm{~K}$. Thus, the total foreground model reads

$$
\begin{aligned}
s_{\mathrm{fg}}(v, p)= & A_{s}(p) g(v)\left(\frac{v}{v_{s}}\right)^{\beta_{s}(p)}+A_{f}(p) g(v)\left(\frac{v}{v_{f}}\right)^{-2.15} \\
& +A_{\mathrm{d}}(p) g(v) \frac{\mathrm{e}^{h v_{\mathrm{d}} / k T_{\mathrm{d}}}-1}{\mathrm{e}^{h v / k T_{\mathrm{d}}}-1}\left(\frac{v}{v_{\mathrm{d}}}\right)^{\beta_{\mathrm{d}}(p)+1} \\
& +m_{v}^{0}+\sum_{i=1}^{3} m_{v}^{i}\left(\hat{\boldsymbol{n}}(p) \cdot \hat{\boldsymbol{e}}_{i}\right)
\end{aligned}
$$

where $g(v)$ is the conversion factor between antenna and thermodynamic temperatures, and $\hat{\boldsymbol{n}}$ is the unit vector of pixel $p$. The free parameters are thus the foreground amplitudes, $A_{\mathrm{s}}, A_{f}$ and $A_{\mathrm{d}}$, and spectral indices, $\beta_{\mathrm{s}}$ and $\beta_{\mathrm{d}}$, for each pixel, and the overall monopole, $m_{v}^{0}$, and dipole amplitudes, $m_{v}^{i}$, for each band. For priors, we adopt the product of the Jeffreys' ignorance prior and an informative Gaussian prior $\left(\beta_{s}=-3 \pm 0.3\right.$ for synchrotron and $\beta_{\mathrm{d}}=1.5 \pm 0.3$ for dust) for the spectral indices, while no constraints are imposed on the amplitudes.

Using the Gibbs (conditional) sampling technique, a set of samples drawn from the posterior distribution. From these samples the marginal posterior mean and rms component maps are derived, as well as the marginal CMB power spectrum posterior distribution.

The code assumes identical beams at all frequencies, and it is therefore necessary to smooth the data to a common resolution, limiting the analysis to large angular scales. For this particular data set, we have chosen a common resolution of $3^{\circ} \mathrm{FWHM}$, with $54^{\prime}$ pixels (Healpix $N_{\text {side }}=64$ ) and with $\ell_{\max }=150$. For more details on the degradation process, see Eriksen et al. (2008). At this resolution, the CPU time for producing one sample is around one wall-clock minute. A total of 5400 samples were produced over four independent Markov chains, of which the first 2400 were rejected due to burn-in. Twelve frequency bands (covering frequencies between 23 and $353 \mathrm{GHz}$ ) were included, for a total cost of around $1000 \mathrm{CPU}$ h.

The main advantage of this approach is simply that it provides us with the exact joint $\mathrm{CMB}$ and foreground posterior distribution for very general foreground models. From this joint posterior distribution, it is trivial to obtain the exact marginal CMB power spectrum and sky signal posterior distributions. Second, since any parametric foreground model may be included in the analysis, the method is very general and flexible. It also provides maps of the posterior means for individual components, and is therefore a true component separation method, and not only a foreground removal tool.

Currently, the main disadvantage of the approach is the assumption of identical beam profiles at each frequency. This strictly limits the analysis to the lowest resolution of a particular data set. However, this is a limitation of the current implementation, and not of the method as such.

\section{A.2. Correlated component analysis (CCA)}

CCA (Bedini et al. 2005) is a semi-blind approach that relies on the second-order statistics of the data to estimate the mixing matrix on sub-patches of the sky. CCA assumes the data model given by Eq. (4), and makes no assumptions about the independence or lack of correlations between pairs of radiation sources. The method exploits the spatial structure of the individual source maps and adopts commonly accepted models for source frequency scalings in order to reduce the number of free parameters to be estimated.

The spatial structures of the maps are accounted for through the covariance matrices at different shifts. From the data model adopted, the data covariance matrices at shifts $(\tau, \psi)$ are given by

$$
\begin{aligned}
\boldsymbol{C}_{\mathrm{d}}(\tau, \psi) & =\left\langle\left[\boldsymbol{d}(\theta, \phi)-\mu_{\mathrm{d}}\right]\left[\boldsymbol{d}(\theta+\tau, \phi+\psi)-\mu_{\mathrm{d}}\right]^{\mathrm{t}}\right\rangle \\
& =\boldsymbol{A} C_{\mathrm{s}}(\tau, \psi) \boldsymbol{A}^{\mathrm{t}}+\boldsymbol{C}_{\mathrm{n}}(\tau, \psi),
\end{aligned}
$$

where $\mu_{\mathrm{d}}$ is the mean data vector, and $(\theta, \phi)$ is the generic pixel index pair. The matrices $\boldsymbol{C}_{\mathrm{d}}(\tau, \psi)$ can be estimated from the data, and the noise covariance matrices $\boldsymbol{C}_{\mathrm{n}}(\tau, \psi)$ are derived from the map-making noise estimations. From Eq. (A.3), we can estimate 
the mixing matrix and free parameters of the source covariance matrices by matching the known quantities to the unknowns, that is by minimizing the following function for $\boldsymbol{A}$ and $\boldsymbol{C}_{\mathrm{s}}(\tau, \psi)$

$$
\sum_{\tau, \psi}\left\|\boldsymbol{A} \boldsymbol{C}_{\mathrm{s}}(\tau, \psi) \boldsymbol{A}^{\mathrm{t}}-\left[\boldsymbol{C}_{\mathrm{d}}(\tau, \psi)-\boldsymbol{C}_{\mathrm{n}}(\tau, \psi)\right]\right\|,
$$

where the Frobenius norm is used and the summation is taken over the set of shift pairs $(\tau, \psi)$ for which data covariances are non-zero. Given an estimate of $\boldsymbol{C}_{\mathrm{s}}$ and $\boldsymbol{C}_{\mathrm{n}}$, Eq. (4) can be inverted and component maps obtained via the standard inversion techniques of Wiener filtering or generalised least square inversion. For the Challenge, harmonic space Wiener filtering was applied, using a mixing matrix obtained by averaging the mixing matrices of different patches. More details on the method can be found in Bonaldi et al. (2006, 2007).

CCA can treat the variability of the spectral properties of each component with the direction of observation by working on sufficiently small sky patches, which must however be large enough to have sufficient constraining power; typically the number of pixels per patch must be around $10^{5}$. To obtain a continuous distribution of the free parameters of the mixing matrix, CCA is applied to a large number of partially overlapping patches.

A drawback of the present version of CCA is common to many pixel-domain approaches to separation: the data must be smoothed to a common resolution. A Fourier-domain implementation of CCA (Bedini \& Salerno 2007) would be able to cope with this problem. Alternatively for the pixel-domain version, the mixing matrix could be estimated from the smoothed maps and then used to separate the sources using the full resolution data.

\section{A.3. Generalised morphological component analysis (GMCA)}

GMCA (Bobin et al. 2007) is a blind source separation method devised for separating sources from instantaneous linear mixtures using the model given by Eq. (4). The components $s$ are assumed to be sparsely represented (i.e. have a few significant samples in a specific basis) in a so-called sparse representation $\boldsymbol{\Phi}$ (typically wavelets). Assuming that the components have a sparse representation in the wavelet domain is equivalent to assuming that most components have a certain spatial regularity. These components and their spectral signatures are then recovered by minimizing the number of significant coefficients in $\mathbf{\Phi}$ :

$\min _{\{\boldsymbol{a}, \boldsymbol{s}\}} \lambda\left\|\boldsymbol{s} \boldsymbol{\Phi}^{T}\right\|+\frac{1}{2}\|\boldsymbol{d}-\boldsymbol{a} s\|_{2}^{2}$.

In Bobin et al. (2007), it was shown that sparsity enhances the diversity between the components thus improving the separation quality. The spectral signatures of $\mathrm{CMB}$ and $\mathrm{SZ}$ are assumed to be known. The spectral signature of the free-free component is approximately known up to a multiplicative constant (power law with fixed spectral index). The synchrotron component is estimated via a separable linear model: $\boldsymbol{d}_{\text {sync }}=a_{\text {sync }} s_{\text {sync }}$ where $a_{\text {sync }}$ is parameterised by a spectral index $\beta_{\text {sync }}$. This spectral index is estimated by solving the following problem:

$$
\min _{\beta}\left\|\boldsymbol{r}_{\text {sync }}-a_{\text {sync }}(\beta) s_{\text {Haslam }}\right\|_{2}^{2}
$$

where $\boldsymbol{r}_{\text {sync }}$ is the residual obtained by extracting the contribution of all the components from the data $\boldsymbol{d}$ except synchrotron. $s_{\text {Haslam }}$ is the Haslam synchrotron map; $a_{\text {sync }}(\beta)$ is the spectral signature of synchrotron emission (power law). More precisely, $\beta$ is estimated such that the Haslam multiplied by $a_{\text {sync }}(\beta)$ matches the residual term $\boldsymbol{r}_{\text {sync }}$.

A Wiener filter is applied to provide the denoised CMB estimate. The main advantage of GMCA is its ability to blindly extract strong galactic emission. Indeed, most galactic emission is well represented in a wavelet basis. The main disadvantage is that it relies on the way the deconvolution of the data is performed: an effective beam is used to account for the convolution.

\section{A.4. Independent component analysis (FastlCA)}

Independent Component Analysis is an approach to component separation, looking for the components which maximise some measure of the statistical independence (Hyvarinen 1999). The FastICA algorithm presented here exploits the fact that nonGaussianity is usually a convenient and robust measure of the statistical independence and therefore it searches for linear combinations $\boldsymbol{y}$ of the input multi-frequency data, which maximise some measure of the non-Gaussianity. In the specific implementation of the idea, employed here, the non-Gaussianity is quantified by the neg-entropy. Denoting by $H(\boldsymbol{y})=-\int p(\boldsymbol{y}) \log p(\boldsymbol{y}) d \boldsymbol{y}$ the entropy associated with the distribution $p$, we define the negentropy as,

$\operatorname{neg}$-entropy $(\boldsymbol{y})=H\left(\boldsymbol{y}_{G}\right)-H(\boldsymbol{y})$,

where $\boldsymbol{y}_{G}$ is a Gaussian variable with the same covariance matrix as $y$. The search for the maxima of the neg-entropy is usually aided by enhancing the role of the higher order moments of $\boldsymbol{y}$, which is achieved by means of a non-linear mapping. In the present implementation, the FastICA finds the extrema of the neg-entropy approximation given by $\left|E[g(\boldsymbol{y})]-E\left[g\left(\boldsymbol{y}_{\mathrm{G}}\right)\right]\right|^{2}$, where $E$ means the average over the pixels, and $g$ represents the non-linear mapping of the data, which may be a power law in the simplest case. The algorithm is straightforwardly implemented in real space, and requires the same angular resolution for all channels. Note that for an experiment like PLANCK where the resolution varies with frequency, this requires smoothing the input data to the lowest resolution before processing. The use of an efficient minimization procedure, with a required number of floating point operations scaling linearly with the size of the data set, makes the computational requirements essentially dominated by memory needed to be allocated to quickly access the multi-frequency data.

The algorithm has been tested so far as a CMB cleaning procedure, because the hypothesis of statistical independence is expected to be verified at least between $C M B$ and diffuse foregrounds. It produced results on real (BEAST, COBE, WMAP) and simulated total intensity data, as well as on polarization simulations, on patches as well as all sky (see Maino et al. 2007, and references therein). The performance is made possible by two contingencies, i.e. the validity of the assumption of statistical independence for $\mathrm{CMB}$ and foregrounds, as well as the high resolution of the present CMB observations, which provides enough of statistical realizations (pixels) for the method to decompose the data into the independent components.

\section{A.5. Harmonic-space maximum entropy method (FastMEM)}

The Maximum Entropy Method (MEM) can be used to separate the CMB signal from astrophysical foregrounds including Galactic synchrotron, dust and free-free emission as well as SZ effects. The particular implementation of MEM used here 
works in the spherical harmonic domain. The separation is performed mode-by-mode allowing a huge optimisation problem to be split into a number of smaller problems. The solution can thus be obtained more rapidly, giving this implementation its name: FastMEM. This approach is described by Hobson et al. $(1998,1999)$ for Fourier modes on flat patches of the sky and by Stolyarov et al. $(2002,2005)$ for the full-sky case.

If we have a model (or hypothesis) $H$ in which the measured data $\boldsymbol{d}$ is a function of an underlying signal $\boldsymbol{s}$, then Bayes' theorem tells us that the posterior probability $\operatorname{Pr}(s \mid d, H)$ is the product of the likelihood $\operatorname{Pr}(\boldsymbol{d} \mid \boldsymbol{s}, H)$ and the prior probability $\operatorname{Pr}(\boldsymbol{s}, H)$, divided by the evidence $\operatorname{Pr}(\boldsymbol{d}, H)$,

$$
\operatorname{Pr}(\boldsymbol{s} \mid \boldsymbol{d}, H)=\frac{\operatorname{Pr}(\boldsymbol{d} \mid \boldsymbol{s}, H) \operatorname{Pr}(\boldsymbol{s} \mid H)}{\operatorname{Pr}(\boldsymbol{d} \mid H)} .
$$

The objective here is to maximise the posterior probability of the signal given the data. Since the evidence in Bayes' theorem is merely a normalisation constant we maximise the product of the likelihood and the prior

$\operatorname{Pr}(\boldsymbol{s} \mid \boldsymbol{d}, H) \propto \operatorname{Pr}(\boldsymbol{d} \mid \boldsymbol{s}, H) \operatorname{Pr}(\boldsymbol{s}, H)$.

We assume that the instrumental noise in each frequency channel is Gaussian-distributed, so that the log-likelihood has a form of a $\chi^{2}$ misfit statistic. We make the assumption that the noise is uncorrelated between spherical harmonic modes. We also assume that the beams are azimuthally symmetric, so that they are fully described by the beam transfer function $B_{\ell}$ in harmonic space. For mode $(\ell, m)$, the log-likelihood is

$\chi^{2}\left(\boldsymbol{s}_{\ell m}\right)=\left(\boldsymbol{d}_{\ell m}-B_{\ell} \boldsymbol{A} \boldsymbol{s}_{\ell m}\right)^{T} N_{\ell m}^{-1}\left(\boldsymbol{d}_{\ell m}-B_{\ell} \boldsymbol{A} \boldsymbol{s}_{\ell m}\right)$

where $\boldsymbol{A}$ is the fixed frequency conversion matrix which describes how the components are mixed to form the data, and $N_{\ell m}^{-1}$ is the inverse noise covariance matrix for this mode. If the instrumental noise is uncorrelated between channels, then this matrix is diagonal. However, unresolved point sources can be modelled as a correlated noise component.

The prior can be Gaussian, and in this case we recover the Wiener filter with the well-known analytical solution for the signal $s$. However, the astrophysical components have strongly nonGaussian distribution, especially in the Galactic plane. Therefore Hobson et al. (1998) suggested that an entropic prior be used instead. In this case, maximising the posterior probability is equivalent to the minimising the following functional for each spherical harmonic mode

$\Phi_{\mathrm{MEM}}\left(\boldsymbol{s}_{\ell m}\right)=\chi^{2}\left(\boldsymbol{s}_{\ell m}\right)-\alpha S\left(\boldsymbol{s}_{\ell m}\right)$

where $S(s)$ is the entropic term, and $\alpha$ is the regularisation parameter. The minimisation can be done numerically using one of a number of algorithms (Press et al. 1992).

FastMEM is a non-blind method, so the spectral behaviour of the components must be known in advance. Since $\boldsymbol{A}$ is fixed, the spectral properties of the components must be the same everywhere on the sky. However, small variations in the spectral properties, for example, dust temperature, synchrotron spectral index or SZ cluster electron temperature, can be accounted for by introducing additional components. These additional components correspond to terms in the Taylor expansion of the frequency spectrum with respect to the relevant parameter.

The initial priors on the components are quite flexible and they can be updated by iterating the component separation, especially if the signal-to-noise is high enough.
It is not necessary for all of the input maps to be at the same resolution since FastMEM solves for the most probable solution for unsmoothed signal, deconvolving and denoising maps simultaneously. It is flexible enough to include any datasets with known window function and noise properties. A mask can easily be applied to the input data (the same mask for all frequency channels) and this does not cause problems with the separation.

Since FastMEM uses priors on the signals, the solution for the signals is biased. This is especially evident if the signal-tonoise ratio is low. It is possible to de-bias the power spectrum statistically, knowing the priors and the FastMEM separation errors per mode. However, one can not de-bias the recovered maps since the errors are quadratic and de-biasing will introduce phase errors in the harmonics.

No information about the input components was used in the separation, and the prior power spectra were based solely on the physical properties of the components and templates available in the literature. The prior on the CMB component was set using the best-fit theoretical spectrum, instead of a WMAP-constrained realisation. This has a significant effect at low multipoles.

\section{A.6. Spectral estimation via expectation-maximization (SEVEM)}

SEVEM (Martínez-González et al. 2003) tries to recover only the CMB signal, treating the rest of the emissions as a generalised noise. As a first step, the cosmological frequency maps, 100, 143 and $217 \mathrm{GHz}$, are foreground cleaned using an internal template fitting technique. Four templates are obtained from the difference of two consecutive frequency channels, which are smoothed down to the same resolution if necessary, to avoid the presence of CMB signal in the templates. In particular, we construct maps of (30-44), (44-70), (545-353) and (857-545) differences. The central frequency channels are then cleaned by subtracting a linear combination of these templates. The coefficients of this combination are obtained minimising the variance of the final clean map outside the considered mask. The second step consists of estimating the power spectrum of the CMB from the three cleaned maps using the method (based on the Expectation-Maximization algorithm) described in Martínez-González et al. (2003), which has been adapted to deal with spherical data. Using simulations of CMB plus noise, processed in the same way as the Challenge data, we obtain the bias and statistical error of the estimated power spectrum and construct an unbiased version of the $C_{\ell}$ 's of the CMB. This unbiased power spectrum is used to recover the CMB map from the three clean channels through Wiener filter in harmonic space. Finally, we estimate the noise per pixel of the reconstructed map using CMB plus noise simulations.

One of the advantages of SEVEM is that it does not need any external data set or need to make any assumptions about the frequency dependence or the power spectra of the foregrounds, other than the fact that they are the dominant contribution at the lowest and highest frequency channels. This makes the method very robust and, therefore, it is expected to perform well for real PLANCK data. Moreover, SEVEM provides a good recovery of the power spectrum up to relatively hig $\ell$ and a small error in the $\mathrm{CMB}$ map reconstruction. In addition the method is very fast, which allows one to characterise the errors of the CMB power spectrum and map using simulations. The cleaning of the data takes around $20 \mathrm{mn}$, while the estimation of the power spectrum and map requires around 15 and $30 \mathrm{mn}$ respectively. In fact, the whole process described, including producing simulations to 
estimate the bias and errors, takes around $30 \mathrm{~h}$ on one single CPU. Regarding weak points, the method reconstructs only the CMB and does not try to recover any other component of the microwave sky although it could be generalised to reconstruct simultaneously the both the CMB and the thermal SZ effect. Also, the reconstructed CMB map is not full-sky, since the method does not aim to remove the strong contamination at the centre of the Galactic plane or at the point source positions. In any case, the masked region excluded for the analysis is relatively small.

\section{A.7. Spectral matching independent component analysis (SMICA)}

The principle of SMICA can be summarised in three steps: 1) compute spectral statistics; 2) fit a component-based model to them; 3) use the result to implement a Wiener filter in harmonic space. More specifically, an idealised operation goes as follows. Denote $\boldsymbol{d}(\xi)$ the column vector whose $i$ th entry contains the observation in direction $\xi$ for the $i$-th channel and denote $\boldsymbol{d}_{\ell m}$ the vector of same size (the number of frequency channels) in harmonic space. This is modelled as the superposition of $C$ components $\boldsymbol{d}_{\ell m}=\sum_{c=1}^{C} \boldsymbol{d}_{\ell m}^{c}$. In Step 1), we compute spectral matrices $\widehat{\boldsymbol{C}}_{\ell}=\frac{1}{2 \ell+1} \sum_{m} \boldsymbol{d}_{\ell m} \boldsymbol{d}_{\ell m}^{\mathrm{T}}$. In Step 2) we model the ensemble-averaged spectral matrix $\boldsymbol{C}_{\ell}=\left\langle\widehat{\boldsymbol{C}}_{\ell}\right\rangle$ as the superposition of $\boldsymbol{C}$ uncorrelated components: $\boldsymbol{C}_{\ell}=\sum_{c=1}^{C} \boldsymbol{C}_{\ell}^{c}$ and, for each component, we postulate a parametric model, that is, we let the matrix set $\left\{\boldsymbol{C}_{\ell}^{c}\right\}_{\ell=0}^{\ell_{\max }}$ be a function of a parameter vector $\theta^{c}$. This parameterization embodies our prior knowledge about a given component. For instance, for the $\mathrm{CMB}$ component, we take $\left[\boldsymbol{C}_{\ell}^{\mathrm{cmb}}\right]_{i j}=e_{i} e_{j} c_{\ell}$ where $e_{i}$ is the known CMB emmission coefficient for channel $i$ and $c_{\ell}$ is the unknown angular power spectrum at frequency $\ell$. The parameter vector for CMB would then be $\theta^{\mathrm{cmb}}=\left\{c_{\ell}\right\}_{\ell=0}^{\ell_{\max }}$. All unknown parameters for all components are then estimated by fitting the model to the spectral statistics, i.e. by solving $\min _{\theta^{1}, \ldots, \theta^{c}} \sum_{\ell=0}^{\ell_{\max }}(2 \ell+1) K\left[\widehat{\boldsymbol{C}}_{\ell} \mid \sum_{c=0}^{C} \boldsymbol{C}_{\ell}^{c}\left(\theta^{c}\right)\right]$ where $K\left[\boldsymbol{C}_{1} \mid \boldsymbol{C}_{2}\right]$ is a measure of mismatch between two covariance matrices $\boldsymbol{C}_{1}$ and $\boldsymbol{C}_{2}$. The resulting values $\hat{\theta}^{1}, \ldots, \hat{\theta}^{C}$ provide estimates $\boldsymbol{C}_{\ell}^{c}\left(\hat{\theta}^{c}\right)$ of $\boldsymbol{C}_{\ell}^{c}$. The Wiener filter estimate of $\boldsymbol{d}_{\ell m}^{c}$ can be expressed as $\hat{\boldsymbol{d}}_{\ell m}^{c}=\boldsymbol{C}_{\ell}^{c} \boldsymbol{C}_{\ell}^{-1} \boldsymbol{d}_{\ell m}$. In practice, we use the fitted spectral matrices estimated at the previous step: component $c$ is estimated as

$\hat{\boldsymbol{d}}_{\ell m}^{c}=\boldsymbol{C}_{\ell}^{c}\left(\hat{\theta}^{c}\right) \boldsymbol{C}_{\ell}(\hat{\theta})^{-1} \boldsymbol{d}_{\ell m}$

and the maps of each component in each channel are finally computed by inverse spherical harmonic transforms.

For processing the current data set, we have used a model containing four components: the $\mathrm{CMB}$, the SZ component, a 4-dimensional Galactic component and a noise component.

The actual processing includes several modifications with respect to this outline: a) beam correction applied to each spectral matrix $\widehat{\boldsymbol{C}}_{\ell}$; b) spectral binning by which the (beam corrected) spectral matrices are averaged over bins of increasing lengths; c) localization implemented via aopdised masks, by which the SMICA process is conducted independently over two different sky zones.

Strengths: a) no prior information used regarding Galactic emission; b) accurate recovery of the CMB via Wiener filtering; c) it is a relatively fast algorithm; d) built-in goodness of fit.

Weaknesses: a) the results reported here do not account for the contribution of point sources for which a convenient model is lacking; b) localization in two zones is probably too crude; c) no separation of Galactic components.

\section{A.8. Wavelet-based high-resolution fitting of internal templates (WI-FIT)}

WI-FIT (Hansen et al. 2006) is based on fitting and subtraction of internal templates. Regular (external) template fitting uses external templates of Galactic components based on observations at frequencies different from the ones used to study the CMB. These templates are fitted to CMB data, the best fit coefficients for each component are found and the templates are subtracted from the map using these coefficients in order to obtain a clean CMB map. WI-FIT differs from this procedure in two respects: (1) it does not rely on external observations of the galaxy but forms templates by taking the difference of CMB maps at different channels. The CMB temperature is equal at different frequencies whereas the Galactic components are not. For this reason, the difference maps contain only a sum of Galactic components. A set of templates are constructed from difference maps based on different combinations of channels; (2) the fitting of the templates are done in wavelet space where the uncertainty on the foreground coefficients is much lower than a similar pixel based approach (in the pixel based approach, no pixel-pixel correlations are taken into account since the correlation matrix will become to large for PLANCK-like data sets. In the wavelet based approach, a large part of these correlations are taken into account in scale-scale covariance matrices).

For calibration purposes, a set of 500 simulated CMB maps need to be produced and the full wavelet fitting procedure applied to all maps. This is where most CPU time goes. For PLANCK resolution maps, around $1 \mathrm{~Gb}$ of memory is necessary to apply WI-FIT and a total of around $400 \mathrm{CPU}$ h are required.

The strength of WI-FIT is that it relies on very few assumptions about the Galactic components. WI-FIT does however assume that the spectral indices do not vary strongly from pixel to pixel within the frequency range used in the analysis. If this assumption is wrong then WI-FIT leaves residuals in the areas where there are strongly varying spectral indices.

Another advantage of WI-FIT is that it is easy to apply and is completely linear, i.e. the resulting map is a linear combination of frequency channels with well known noise and beam properties. This will in general result in increased noise variance in the cleaned map. In order to avoid this, we smooth the internal templates in order to make the noise at small scales negligable and at the same time not make significant changes to the shape of the diffuse foregrounds. If the diffuse foregrounds turn out to be important at small scales $l>300$, the smoothing of the internal templates will significantly reduce the ability of WI-FIT to perform foreground cleaning at these scales. Tests on the WMAP data have shown that diffuse foregrounds do not seem to play an important role at such small scales. This is valid for the frequency range observed by WMAP (i.e. at LFI-frequencies), similar tests will need to be made for the PLANCK HFI data.

Finally, WI-FIT does not do anything to the point sources, which need to be masked.

\section{References}

Ashdown, M. A. J., Baccigalupi, C., Balbi, A., et al. 2007, A\&A, 467, 761 Bedini, L., \& Salerno, E. 2007, Lect. Notes Artif. Intell., 4694, 9 Bedini, L., Herranz, D., Salerno, E., et al. 2005, EURASIP J. Appl. Sig. Proc., 15,2400

Bennett, C. L., Halpern, M., Hinshaw, G., et al. 2003a, ApJS, 148, 1

Bennett, C. L., Hill, R. S., Hinshaw, G., et al. 2003b, ApJS, 148, 97 Bertin, E., \& Arnouts, S. 1996, A\&AS, 117, 393

Bobin, J., Starck, J.-L., Fadili, J., \& Moudden, Y. 2007, IEEE Trans. Image Processing, 16, 2662 
Bonaldi, A., Bedini, L., Salerno, E., Baccigalupi, C., \& de Zotti, G. 2006, MNRAS, 373, 271

Bonaldi, A., Ricciardi, S., Leach, S., et al. 2007, MNRAS, 382, 1791

Bouchet, F. R., \& Gispert, R. 1999, New Astron., 4, 443

Cardoso, J.-F., Martin, M., Delabrouille, J., Betoule, M., \& Patanchon, G. 2008, [arXiv:0803.1814]

Colafrancesco, S., Mazzotta, P., Rephaeli, Y., \& Vittorio, N. 1997, ApJ, 479, 1

Davies, R. D., Dickinson, C., Banday, A. J., et al. 2006, MNRAS, 370, 1125

de Zotti, G., Ricci, R., Mesa, D., et al. 2005, A\&A, 431, 893

Delabrouille, J., Cardoso, J.-F., \& Patanchon, G. 2003, MNRAS, 346, 1089

Dickinson, C., Davies, R. D., \& Davis, R. J. 2003, MNRAS, 341, 369

Dunkley, J., Komatsu, E., Nolta, M. R., et al. 2008 [arXiv: 0803. 0586]

Eriksen, H. K., Jewell, J. B., Dickinson, C., et al. 2008, ApJ, 676, 10

Finkbeiner, D. P., Davis, M., \& Schlegel, D. J. 1999, ApJ, 524, 867

Gawiser, E., Finkbeiner, D., Jaffe, A., et al. 1998, [arXiv: astro-ph/9812237]

Gold, B., Bennett, C. L., Hill, R. S., et al. 2008, [arXiv:0803.0715]

González-Nuevo, J., Argüeso, F., López-Caniego, M., et al. 2006, MNRAS, 369, 1603

González-Nuevo, J., Massardi, M., Argüeso, F., et al. 2008, MNRAS, 384, 711

Górski, K. M., Hivon, E., Banday, A. J., et al. 2005, ApJ, 622, 759

Granato, G. L., De Zotti, G., Silva, L., Bressan, A., \& Danese, L. 2004, ApJ, 600,580

Hansen, F. K., Banday, A. J., Eriksen, H. K., Gorski, K. M., \& Lilje, P. B. 2006 , Astrophys. J., 648, 784

Haslam, C. G. T., Salter, C. J., Stoffel, H., \& Wilson, W. E. 1982, A\&AS, 47,1

Herranz, D., Sanz, J. L., Hobson, M. P., et al. 2002, MNRAS, 336, 1057

Hinshaw, G., Spergel, D. N., Verde, L., et al. 2003, ApJS, 148, 135

Hinshaw, G., Nolta, M. R., Bennett, C. L., et al. 2007, ApJS, 170, 288

Hinshaw, G., Weiland, J. L., Hill, R. S., et al. 2008, [arXiv:0803.0732]

Hobson, M. P., Jones, A. W., Lasenby, A. N., \& Bouchet, F. R. 1998, MNRAS, 300, 1

Hobson, M. P., Barreiro, R. B., Toffolatti, L., et al. 1999, MNRAS, 306, 232

Huffenberger, K. M., Eriksen, H. K., Hansen, F. K., Banday, A. J., \& Gorski, K. M. 2007
Hyvarinen, A. 1999, IEEE Signal Proc. Lett. 6, 6, 145

Jones, W. C., Ade, P. A. R., Bock, J. J., et al. 2006, ApJ, 647, 823

Komatsu, E., Kogut, A., Nolta, M. R., et al. 2003, ApJS, 148, 119

López-Caniego, M., Herranz, D., González-Nuevo, J., et al. 2006, MNRAS, 370, 2047

López-Caniego, M., González-Nuevo, J., Herranz, D., et al. 2007, ApJS, 170, 108

Maino, D., Farusi, A., Baccigalupi, C., et al. 2002, MNRAS, 334, 53

Maino, D., Donzelli, S., Banday, A. J., Stivoli, F., \& Baccigalupi, C. 2007, MNRAS, 374, 1207

Martínez-González, E., Diego, J. M., Vielva, P., \& Silk, J. 2003, MNRAS, 345, 1101

Masi, S., Ade, P. A. R., Bock, J. J., et al. 2006, A\&A, 458, 687

Melin, J.-B., Bartlett, J. G., \& Delabrouille, J. 2006, A\&A, 459, 341

Miville-Deschênes, M.-A., Lagache, G., Boulanger, F., \& Puget, J.-L. 2007, A\&A, 469, 595

Miville-Deschênes, M., Ysard, N., Lavabre, A., et al. 2008, ArXiv e-prints, 802 Negrello, M., Magliocchetti, M., Moscardini, L., et al. 2004, MNRAS, 352, 493 Poutanen, T., de Gasperis, G., Hivon, E., et al. 2006, A\&A, 449, 1311

Press, W. H., Teukolsky, S. A., Vetterling, W. T., \& Flannery, B. P. 1992, Numerical recipes in FORTRAN. The art of scientific computing (Cambridge: University Press), 2nd edn.

Reinecke, M., Dolag, K., Hell, R., Bartelmann, M., \& Enßlin, T. A. 2006, A\&A, 445,373

Schlegel, D. J., Finkbeiner, D. P., \& Davis, M. 1998, ApJ, 500, 525

Serjeant, S., \& Harrison, D. 2005, MNRAS, 356, 192

Stolyarov, V., Hobson, M. P., Ashdown, M. A. J., \& Lasenby, A. N. 2002, MNRAS, 336, 97

Stolyarov, V., Hobson, M. P., Lasenby, A. N., \& Barreiro, R. B. 2005, MNRAS, 357,145

Tegmark, M., Eisenstein, D. J., Hu, W., \& de Oliveira-Costa, A. 2000, ApJ, 530, 133

The Planck Collaboration 2005, ESA-SCI(2005), [arXiv:astro-ph/0604069]

Wright, E. L., Chen, X., Odegard, N., et al. 2008, [arXiv: 0803.0507] 